\title{
2 Solid-Phase Microextraction and Related Techniques
}

\section{Habib Bagheri*, Hamed Piri-Moghadam, Mehrnoush Naderi, Ali Es'haghi and Ali Roostaie}

Environmental and Bio-Analytical Laboratories, Department of Chemistry, Sharif University of Technology, P.O. Box 11365-9516, Tehran, Iran

*e-mail address: bagheri@sharif.edu

\subsection{Introduction}

Solid-phase microextraction (SPME) is a rapid, inexpensive and solventless extraction technique for the isolation and preconcentration of solutes from liquid or gaseous matrices. SPME has several important advantages compared to the traditional sample preparation techniques:

1. It is a rapid, simple, solvent free and sensitive method for the extraction of analytes.

2. It is a simple and effective adsorption/desorption technique.

3. It is compatible with a wide range of analytical separation and detection techniques.

4. It provides linear results for wide concentrations of analytes.

5. It has small size, which is convenient for designing portable devices for field sampling.

6. It gives highly consistent, quantifiable results from very low concentrations of analytes.

\subsection{Solid-phase Microextraction Fundamentals}

\subsubsection{Principle of Solid-phase Microextraction}

For a two-phase system, the amount absorbed by the coating at equilibrium can be calculated using equation 2.1 .

$n=V_{1} V_{2} K C_{0} /\left(K V_{1}+V_{2}\right)$

where $n$ is the mass absorbed by the coating, $V_{1}$ and $V_{2}$ are the respective volumes of the coating and the aqueous solution, $K$ is the partition coefficient of the analyte between the coating and the sample, and $C_{0}$ is the initial concentration of the analyte in the aqueous solution (Louch et al., 1992). The mass adsorbed depends on the distri- 
bution constant and the volume of the coating phase. A linear relationship is observed between the amount of analyte adsorbed and its concentration in the solution. With a three-phase system, a small amount of analyte is present in the headspace. When its volume is insignificant or when the solutes are scarcely volatile, the mass of solute adsorbed by the coating can be expressed by equation 2.1 (Rivasseau \& Caude, 1995).

In a three phase system during SPME, the rules based on the equilibrium between various phases can be employed. The equilibrium constants of analyte between each two phases can be written in equation 2.2:

$K_{f w}=\frac{C_{f}}{C_{w}} ; \quad K_{h w}=\frac{C_{h}}{C_{w}} ; \quad K_{f h}=\frac{C_{f}}{C_{h}}$

where $C_{f}$ is the equilibrium concentration of the analyte in the fiber coating, $C_{w}$ is the equilibrium concentration of the analyte in the aqueous medium and $C_{h}$ is the equilibrium concentration of the analyte in the headspace. The molar concentrations can be replaced by the number of analyte molecules in the corresponding phase volume. Also, considering that $n_{f}$ is the number of molecules in the fiber and $n_{0}$, initial number of molecules in the aqueous phase, and replacing their corresponding equilibrium constants and phase volumes, $n_{f}$ can be written:

$n_{f}=\frac{K_{f h} K_{h w} V_{f} n_{0}}{K_{f h} K_{h w} V_{f}+K_{h w} V_{h} V_{w}}$

where $V_{w}$ is the volume of aqueous phase, $V_{f}$ is the volume of coating and $V_{h}$ is the volume of the sample headspace. Considering the Henry's law constants, it would be possible to reach to the following equation:

$n_{f}=\frac{K_{f h} V_{f} n_{0}}{K_{f h} V_{f}+K_{h w} V_{h}+V_{w}}$

A linear relationship between $n_{f}$ and $n_{0}$ can be derived from equation 2.4. However, due to the fact that SPME is an equilibrium-based technique, the maximum recovery of SPME in equilibrium, $R_{\max }$, can be defined:

$R_{\max }=\frac{n_{f}}{n_{0}}=\frac{K_{f w} V_{f}}{K_{f w} V_{f}+K_{h w} V_{h}+V_{w}}$

The increase of the ratio of liquid to headspace volumes lightly improves the efficiency of extraction with any size fiber coating (Bagheri et al., 2005a). Considering equation 2.5, an increase in the headspace volume $\left(V_{h}\right)$ accompanied by a decrease of the solution volume $\left(V_{w}\right)$ in a defined system results in lower sorbed mass $(n)$ on the fiber. The headspace volume is supposed to be kept to a minimum because in case of $V_{h}<V_{f}$ sampling from the headspace does not affect the amount of analyte sorbed by the coating (Zhang \& Pawliszyn, 1993). It is not necessary to increase the entire volume of sampling vials because the efficiency of the extraction is presumably not enhanced if the relative volumes of liquid and headspace remain the same 
(Penton, 1997). Although the principle behind SPME is based on an equilibrated partition process, it is not essential to wait for a full equilibrium to be reached. As long as the extraction time is standardized, it is be possible to obtain reproducible and sufficiently sensitive analysis. Of course, for the sake of acceptable repeatability it is rather necessary to choose equilibrium time in the region where the least possible changes on the analytical signal could be observed (Havenga \& Rohwer, 1999).

\subsubsection{Different Modes of Solid-phase Microextraction}

It is possible to perform the SPME procedure under different configurations (Figure 2.1), categorized into two basic modes of diffusion mediated by stirring and diffusion mediated by flow through. The extracting phase could be coated, either physically or chemically, on the fiber, stir bar, thin film or on the inner surface of a tip, syringe and tube.

\subsubsection{Coupling to Analytical Instrumentation}

SPME can be coupled to different analytical instruments depending on the mode of operation and can be performed via both off-line and on-line configuration. The on-line combination may include the on-line extraction-desorption processes or just on-line desorption of the target analytes. Fiber-based SPME is directly coupled to gas chromatography (GC) for on-line desorption and through an appropriate desorption chamber to high performance liquid chromatography (HPLC). In the mean time, capillary microextraction (CME) is directly coupled to HPLC for extraction-desorption and its on-line combination to GC is also feasible via an appropriate interface. The other SPME-related techniques are usually employed in off-line combination with GC and HPLC.

\subsubsection{Off-line Coupling}

In off-line mode, the SPME extracting probe is inserted into an appropriate solvent to desorb the trapped analytes from the relevant probe. After complete desorption of analytes, the solvent containing analytes is subsequently exposed to a stream of nitrogen gas for desolvation and followed by reconstitution in a few microliter of an appropriate solvent. Eventually, it is injected into the appropriate analytical instrument.

\subsubsection{On-line Coupling}

Off-line SPME often suffers from the loss of analytes during the evaporation step and therefore lack of accuracy. To overcome this problem, on-line configuration is a very suitable alternative. Apart from the higher accuracy and precision of the on-line SPME 


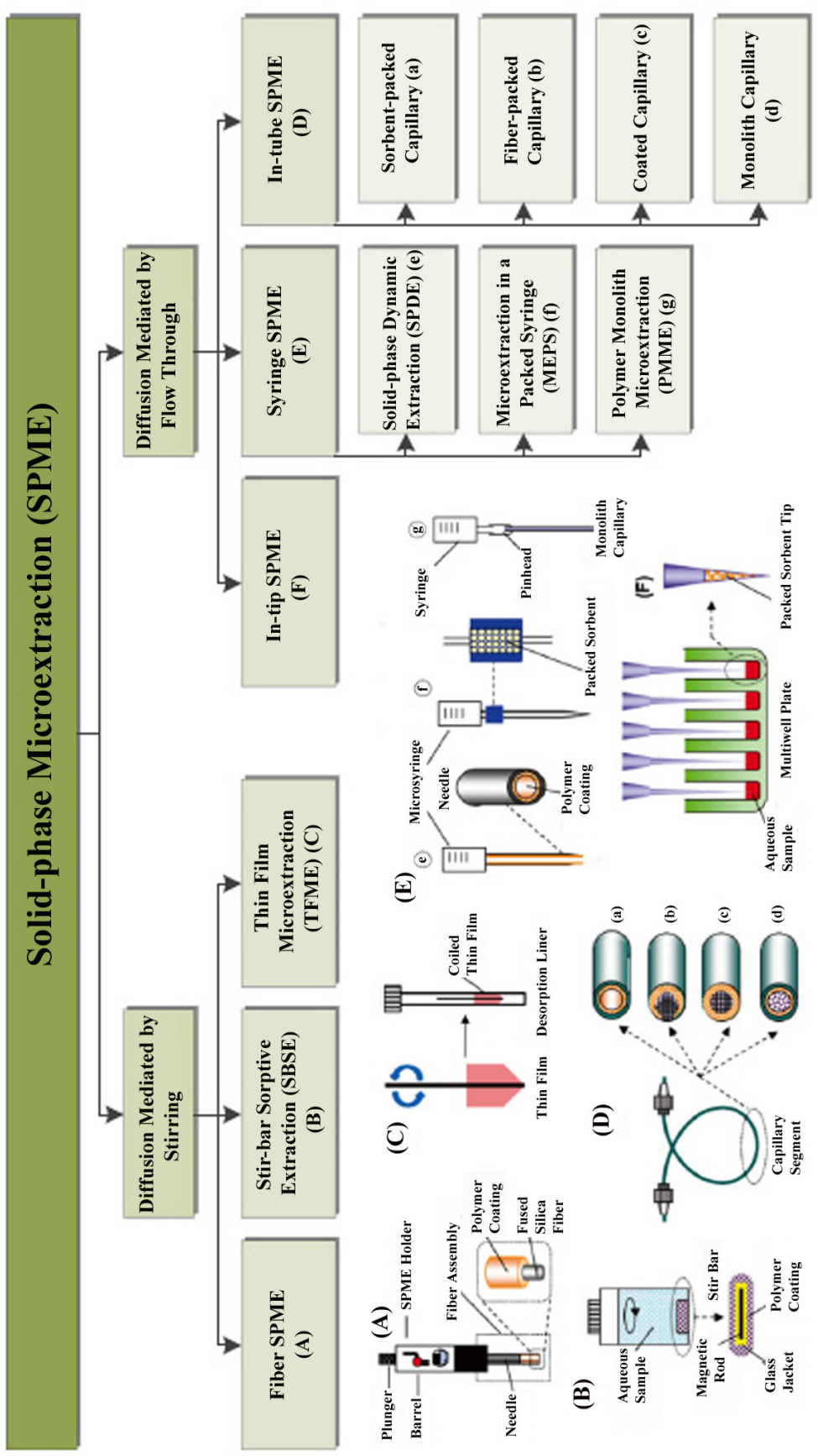

Figure 2.1 Schematic diagram for different modes of SPME. Reprinted from Kabir et al. (2013) with permission from Elsevier. 


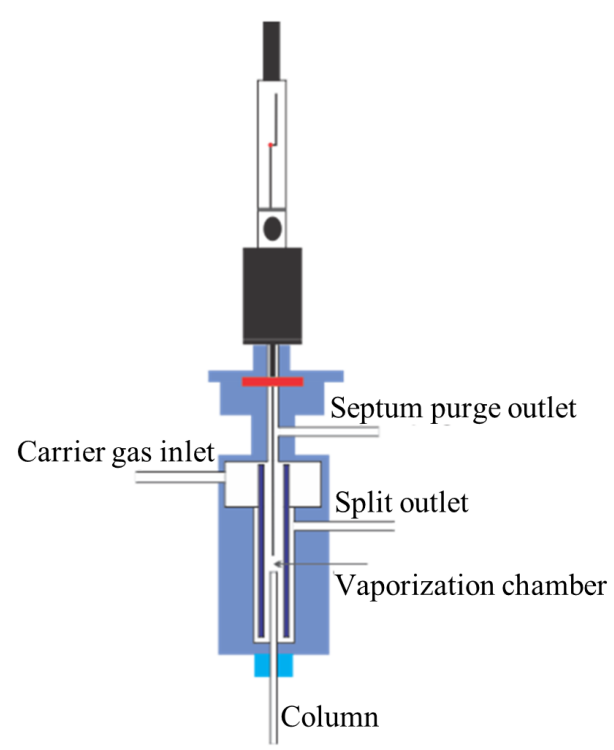

Figure 2.2 On-line desorption of fiber SPME in the injection port of GC.

set up, most of the time it is favored as far as automation and solvent consumption issues are concerned. On the other hand, CME could be directly coupled to analytical instruments using an on-line set up for extraction-desorption of the target analytes while fiber SPME needs an appropriate devoted interface containing a desorption chamber in combination with HPLC. While direct on-line coupling of CME and HPLC is mostly common, GC is also frequently used for on-line desorption of fiber SPME.

\subsection{On-line Coupling to Gas Chromatography}

Fiber SPME: In on-line SPME, desorption of analytes should be performed as rapidly as possible to avoid any possible peak broadening. In GC, the rapid desorption happens inside the high temperature injection port and the fiber SPME introduction is quite like standard injection syringe. The plunger movement allows exposure of the fiber during extraction and desorption and its protection in the needle during storage and penetration of the septum (Figure 2.2). When the desorption temperature of a GC is kept at high temperature and fibers with thin coatings are used, it would be expected that all the analytes are in the gas phase as soon as the fiber coating is inserted in the injection port. Under these circumstances, the sample desorption time is very much dependent on the temperature of the GC injection compartment (Lord \& Pawliszyn, 2000).

CME: A schematic diagram for on-line coupling of CME to GC through an interface is shown in Figure 2.3. The ambient air, solution, or solution headspace is sampled by passing a gas or liquid through the open capillary. The analytes are trapped on the coating of a short piece of a capillary GC column or a prepared sorbent on the 


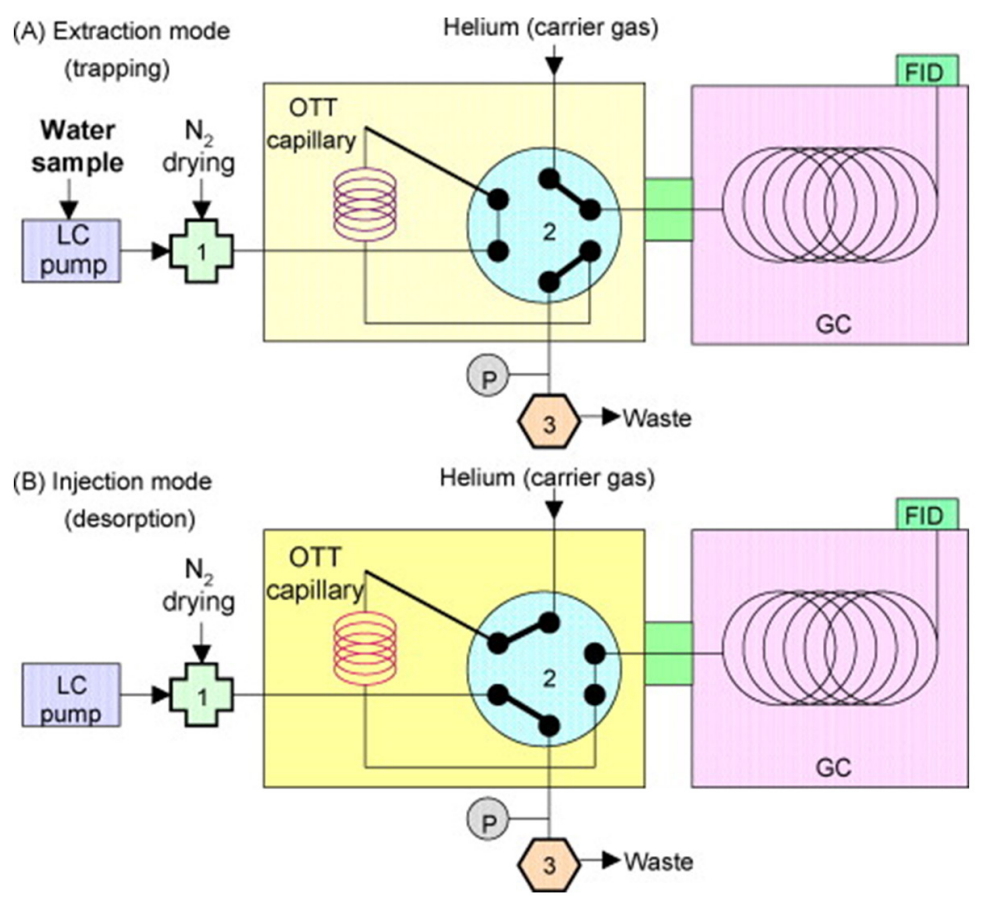

Figure 2.3 Schematic diagram of on-line CME-GC (A) Extraction and (B) Desorption. Reprinted from Kataoka et al. (2009) with permission from Elsevier.

inner surface of a capillary tube. The analytes are retained as they pass through the capillary tubing containing the stationary phase and they are subsequently desorbed, either by solvent or by thermal desorption (TD). The sample is forced to flow through the capillary and the analytes pass the trapping medium which has been coated onto the walls by diffusion. The sufficient thermal stability of GC stationary phases allows collected analytes to be thermally desorbed from the coating material after sampling. These analytes can be desorbed directly onto a GC column for analysis, avoiding dilution of the sample with solvent (Kataoka et al., 2009).

The possibility of sample cross-contamination and degradation are reduced as intermediate sample handling steps are eliminated. Another advantage is that even very volatile compounds can be enriched at ambient temperature, therefore, the need for a cryogenic refocusing step is unnecessary.

\subsection{On-line Coupling to Liquid Chromatography}

On-line combination of SPME fibers and CME to HPLC benefits from automation of the extraction. In HPLC, rapid desorption is achievable using a mobile phase with strong eluting power. Thus, the mobile phase composition should provide complete 


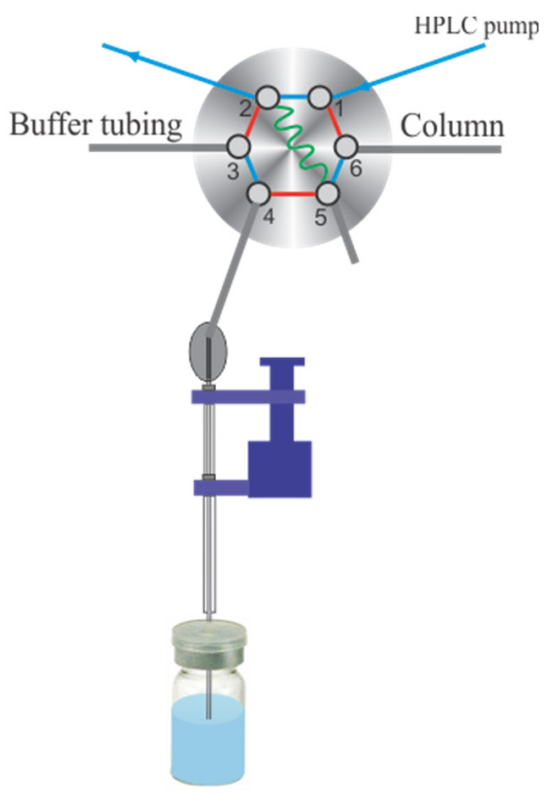

Figure 2.4 On-line extraction/desorption of fiber SPME to HPLC via an interface.

desorption of the extracted analytes, while the proper separation of the analytes in the analytical column can be performed.

Fiber SPME: Fiber SPME could be employed in on-line mode with HPLC in which a desorption chamber is required. Figure 2.4 shows the appropriate configuration for this setup (Lord \& Pawliszyn, 2000).

CME: According to Figure 2.5, extraction is performed by passing the spiked aqueous samples through the tube. After extraction, the HPLC mobile phase is used for on-line desorption and elution of the extracted analytes from the tube to the HPLC column (Bagheri et al., 2011c, 2012d).

\subsection{Extractant Phases in Solid-phase Microextraction}

\subsubsection{Conventional Extractant Phases}

Selection of the extracting phase is the most important step governing the selectivity of SPME. In the original SPME, the thermally activated polyimide film and uncoated fused silica were used as the extracting phases. The polyimide had to be treated at $350{ }^{\circ} \mathrm{C}$ prior to the extraction. Both could be applied to the determination of volatile chlorinated organic compounds in water. Benzene, toluene, ethylbenzene, and 
Load position

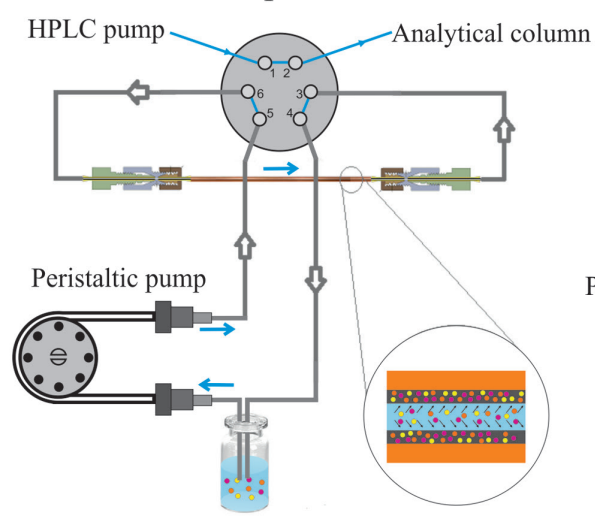

Inject position

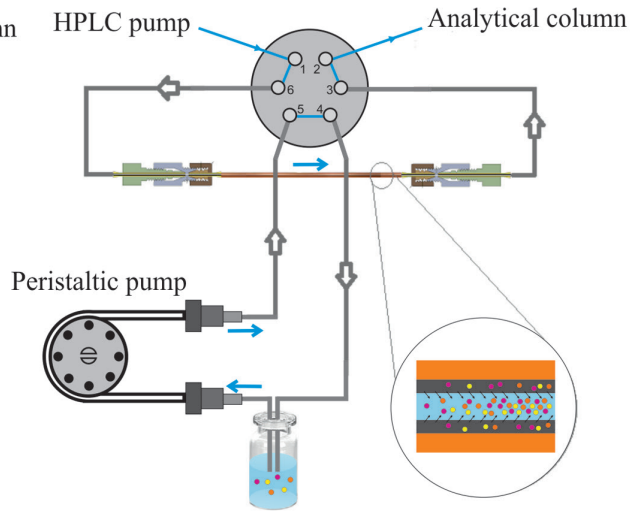

Figure 2.5 On-line extraction/desorption using CME-HPLC.

xylenes (BTEX) were also trapped on the bare silica fiber. Several types of coatings including polydimethylsiloxane (PDMS), polyacrylate (PA), polydimethylsiloxane/ divinylbenzene (DVB), Carboxen/PDMS, Carbowax (CW)/template resin fibers have become commercially available. Among those, PDMS and PA coatings are the most well-studied and characterized. For a specific application, the coating is chosen based on the polarity of the analyte (Zhang et al., 1994). PDMS is less polar than PA; thus it is widely used for the extraction of non-polar compounds such as substituted benzenes (Potter \& Pawliszyn, 1992; MacGillivray et al., 1994) and polyaromatic hydrocarbons (PAHs) (Potter \& Pawliszyn, 1994). For polar compounds such as ketones and alcohols, polar coatings like PA and CW are preferred. The selectivity difference of polar and nonpolar coatings has been studied by analyzing organophosphorus insecticides (Magdic et al., 1996; Valor et al., 1997). Among the organophosphorus compounds tested, triazophos and methylparathion showed greater affinity towards PA than PDMS, while diazinon and prothiofos exhibited comparable or even higher affinity towards PDMS. Volatile aldehydes, pyrazines, pyridines and thiazoles were extracted by PDMS and CW/DVB. Although these fibers show different selectivity to different groups of compounds, both fibers can extract solutes with more alkyl substitutions better for all four groups of compounds. These results coincide with results obtained for some pesticides, including $N$-substituted amines, $N$-heterocyclic compounds and organophosphorus compounds. These compounds were extracted using the PA fiber coating (Eisert \& Levsen, 1995). A linear correlation between extraction efficiency (relative peak response after SPME to the standard chromatogram) and the octanol-water partition coefficient for some triazine pesticides was obtained. 
Despite numerous and pronounced advantages of SPME over traditional sample preparation techniques, the conventional fiber SPME suffers from significant shortcomings, which include:

1. Relatively low operating temperature due to poor thermal stability of the physically held sorbent coating;

2. Instability and swelling of the fiber coatings in organic solvents;

3. Fragility of the coated fibers;

4. Mechanical instability of the substrates;

5. Bending of the SPME syringe needle during operation;

6. Low sorbent loading that results in poor extraction sensitivity;

7. Pronounced run-to-run and batch-to-batch variability;

8. Relatively long extraction equilibrium time due to the slow diffusion of analytes into pure organic polymers used as the extracting medium;

9. Limited numbers of commercially available fiber coatings;

10. Short lifetime of the physically held sorbent coating.

Many modifications have been implemented to circumvent these problems, including the fabrication of metal-based substrates to enhance the mechanical instability of the fibers and adaption of new methodologies to improve the thermal and solvent stability of the coatings. Methodologies based on sol-gel technique, conductive polymers, nanomaterials, nanocomposites, the usage of an electrospinning system and preparation of selective sorbents by molecularly imprinted polymers (MIPs) and molecularly imprinted xerogels (MIXs) are among these efforts.

\subsubsection{Extractant Phases Based on Inorganic Polymerization}

Although the use of SPME fibers is increasingly popular, their relatively low recommended operating temperature $\left(\sim 240-280{ }^{\circ} \mathrm{C}\right)$, their instability and swelling in organic solvents (greatly restricting their use with HPLC), fiber breakage and stripping of coatings are obstacles to the analysis of some chemicals. Apart from the fiber breakage, other limitations are due to the physical interactions between the extracting phase and solid substrates. Low operating temperature and stripping of coatings affects the performance and lifetimes of the fibers. Preparation of chemically bonded SPME fibers has been the center of attention for many researchers to overcome some of these problems.

Inorganic polymerization, better known as the 'sol-gel process', is a general method for preparing oxides by the 'wet route' (i.e., in solution) at room temperature. The sol-gel process was discovered by Ebelmann $(1846,1847)$ and has benefited from developments of inorganic polymerization.

Inorganic polymerization has experienced an extraordinary revival during the last two decades, as it corresponds to a general method for the preparation of oxides 

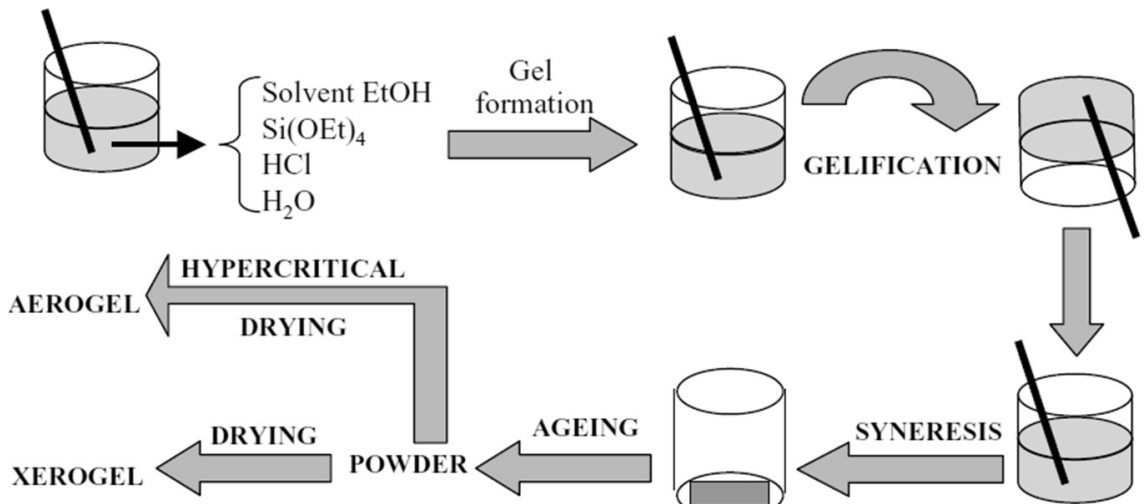

POWDER

AGEING
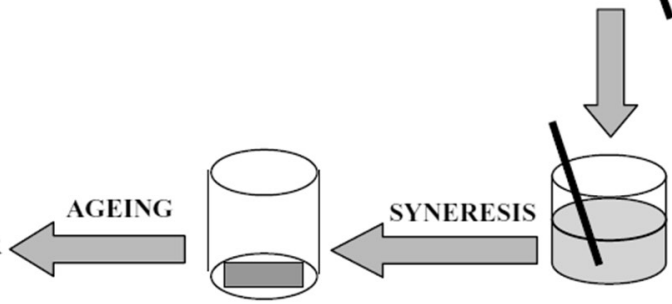

PHASE DEMIXING

Figure 2.6 Schematic diagram of the different steps of the sol-gel process. Reprinted from Corriu \& Trong Anh (2009) with permission from John Wiley \& Sons.

under mild conditions. Reaction with water at room temperature and in the presence of a (acidic, basic or nucleophilic) catalyst, of a $\mathrm{Si}(\mathrm{OR})_{4}$ (R: Me, Et, iPr, etc.) solution gives a transparent solid occupying the entire volume. This solid is not a precipitate, but a gel enclosing the solvent and sticking to the walls of the recipient. Figure 2.6 shows the various steps of the process (Corriu \& Trong Anh, 2009).

After a lapse of time which varies with the experimental conditions, a phase demixing occurs in which the solid $\mathrm{SiO}_{2}$ expels the solvent and becomes denser. This stage is called syneresis. The solid obtained is ground, washed and vacuum dried to give a xerogel. Other treatments are possible, for instance, hypercritical drying yields aerogels, which have valuable dielectrical and insulating properties. In spite of the interesting nature of these treatments, we shall focus on the xerogels, which are mostly used in the field of nanomaterials (Corriu \& Trong Anh, 2009).

In 1997, sol-gel technology was used for the preparation of sorbents in SPME by Malik and co-workers (Chong et al., 1997; Wang et al., 1997). It can be an excellent alternative to conventional coating preparation methods due to its inherent advantageous features and performances. These include single step manufacturing process, material homogeneity at the molecular level, chemical bonding between the sorbent and the fused silica surface, high thermal and solvent stability and the porous structure of the hybrid material. Moreover, sol-gel organic-inorganic hybrid materials provide desirable sorptive properties that are difficult to achieve using either purely organic or inorganic material. Precursor, coating polymer, catalyst and deactivating agent (non polar fibers) are required for preparation of sol-gel based SPME fibers. Moreover, functionality of the solid support is another major issue. The research concerning the preparation of new SPME fibers includes three sol-gel based features of 
selecting (i) precursors, (ii) coating polymers and (iii) modifiers to achieve fibers with specific physical capabilities and the desired polarity and selectivity (Bagheri et al., 2012e). For the preparation of a sol-gel coating in SPME, the presence of a precursor is necessary, and the addition of the coating polymer and/or modifier could be influential to improve some major interactions and available porosity.

\subsubsection{Preparation of Sorbents by Sole Precursor}

Preparation of sorbent by a sole precursor in water using an acid-catalyzed process is also possible. In this regard, a selected precursor should contain an appropriate organic group, an alkyl alkoxysilane, to prepare organically modified silica (ORMOSIL). ORMOSIL should contain appropriate groups to interact with desired analytes via mechanisms such as van der Waals, dipole-dipole and hydrogen bonding. A typical sol-gel processing is shown in Figure 2.7A.

In practice, the sol-gel process is, considering the macroscopic scale, very simple. In one step and at room temperature, it transforms a molecule into a material ready for shaping. At the nanoscopic and microscopic scales, it is in fact a very complex process consisting of several transformations of very different natures involving three distinguishable states of matter: solution, colloid and solid.

The first state occurs in solution and corresponds to the formation of the $\mathrm{Si}-\mathrm{O}-\mathrm{Si}$ bonds. At first, the precursor gives rise to nanometric linear and cyclic oligomers which, by polycondensation reactions, gradually become intermingled to yield crosslinked polymers (10-100 $\mathrm{nm}$ in size). These polymers aggregate into micrometer-sized colloids, which are detectable by light diffusion. This colloidal solution is a viscous sol and corresponds to a crucial phase of the process (Figure 2.8). It is the viscous sol which permits the shaping of the material. It can be drawn into fibers, molded as a bulk solid or used as a covering by dip or spin coating (Corriu \& Trong Anh, 2009).

In the second stage, the sol is solidified into a gel, a process known as sol-gel transition. This step corresponds to a chemical reaction occurring at the surface of the colloids. The rapid gelification can be easily understood: a few chemical bonds between the voluminous colloids, whose surfaces are covered with $\mathrm{SiOH}$ (or SiOR) groups, are enough to create a solid wide-meshed network capable of retaining all the solvent and of occupying all the space.

The next step corresponds to a solid phase development. The number of bonds between the colloids increases, thus speeding up the densification of the solid. The initial stage, called syneresis, corresponds to the expulsion of the solvent. Continuation of this bonding process in the solid phase leads to an amorphous solid having stabilized granulometry and porosity, which depend on the experimental conditions. This is called the Oswald ageing process. It is very important because it controls the macroscopic characteristics of the solid. The final step consists of the drying of the washed solid and it should be noted that the drying conditions can significantly affect the material's texture (Corriu \& Trong Anh, 2009). 

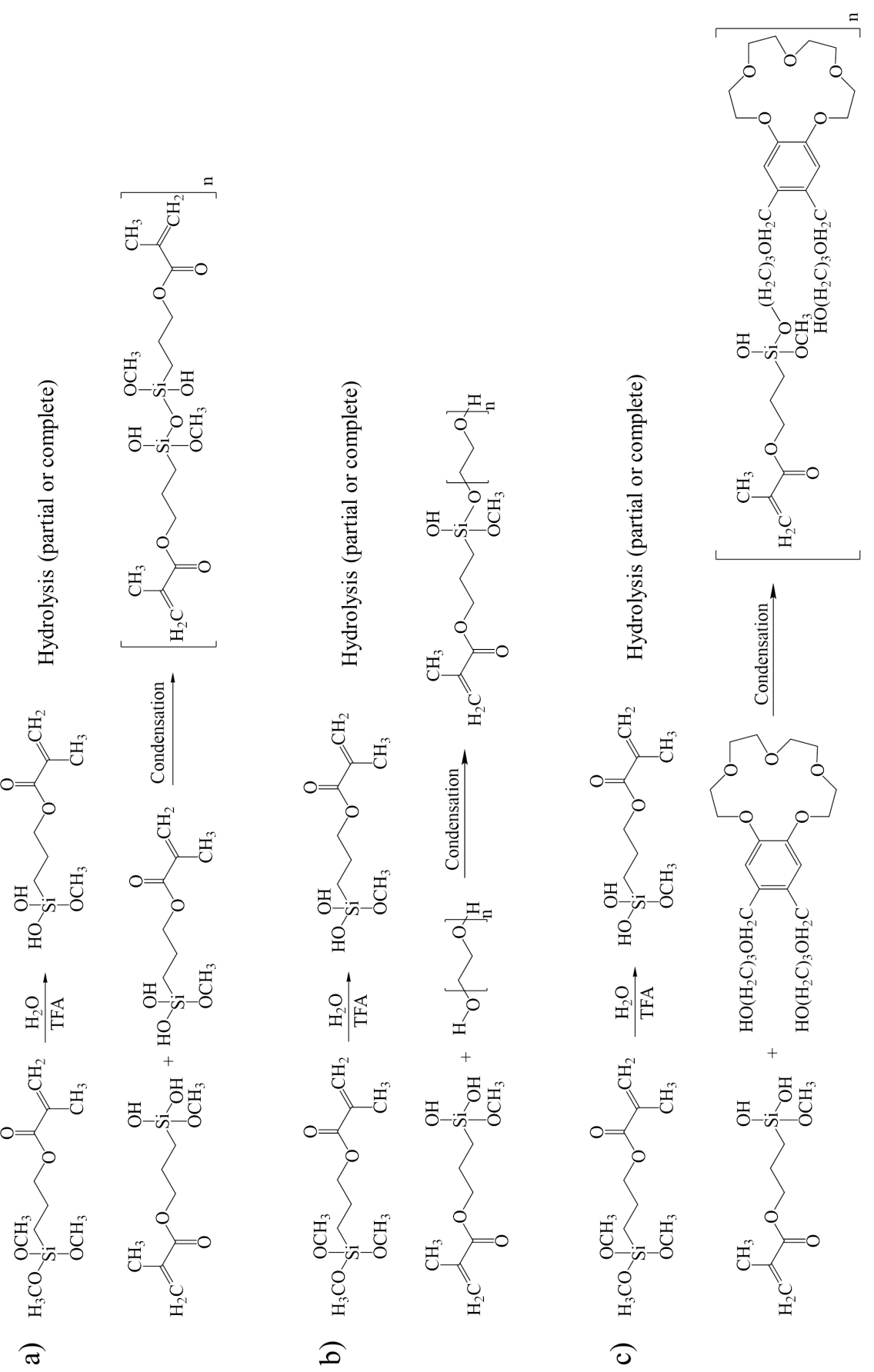

Figure 2.7 Preparation of SPME sorbents by (A) sole precursor, (B) precursor and coating polymer (C) precursor and a modifier. 


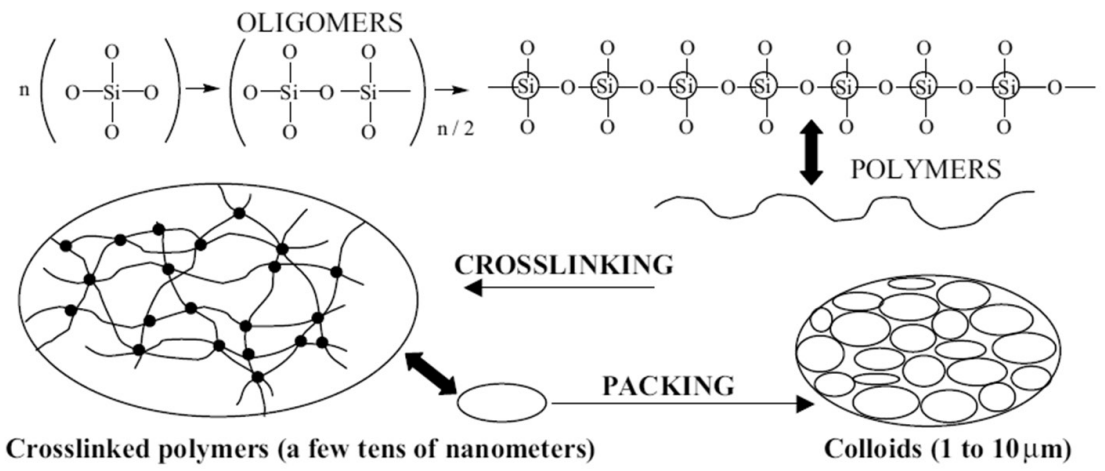

Figure 2.8 Schematic diagram for the formation of oligomers. Reprinted from Corriu \& Trong Anh (2009) with permission from John Wiley \& Sons.

\subsubsection{Preparation of Sorbents by Precursor and Coating Polymer}

One of the advantages of the sol-gel technique is the possibility to prepare multi-component materials which favorably affect the porosity and enhance interactions with the desired analytes through the addition of polymers into the xerogel (sorbent) structure. Addition of polyethylene glycol to a precursor is shown in Figure 2.7B.

\subsubsection{Preparation of Sorbents by Precursor and a Modifier}

One of the most important advantages of the sol-gel route is the possibility of designing the material structure and properties through proper selection of the sol-gel precursor and other building blocks. Different modifiers such as crown ethers (Yu et al., 2004), calixarenes (Li et al., 2004), carbon nanotubes (CNTs) (Wang et al., 2006; Sarafraz-Yazdi et al., 2010a, 2010b; Bagheri et al., 2011a), fullerene (Yu et al., 2002) and $\beta$-cyclodextrin (Zhou et al., 2007) have been used to enhance the extraction efficiency of the prepared sorbents. Sol-gel chemistry is widely used for the preparation of materials suitable for a wide range of applications. This technology has been used for preparing SPME fiber coatings and exhibit many advantages by providing efficient incorporation of organic component into the inorganic polymeric structure in solution under very mild thermal conditions. Many inherent advantages of sol-gel technology are their high thermal stability, porous structure, high degree of flexibility in coating composition, by varying the proportion of the sol solution ingredients or using a deactivation reagent. In this technique precursors are mixed at the molecular level and multicomponent materials could be formed at much lower temperature than traditional processing method. The guest molecules could be introduced by dissolving or suspending them in the hydrolyzates, without harming their physical properties. A typical reaction for this type of mechanism is shown in Figure 2.7C. 
Calixarenes are regarded as the third generation of supramolecules next to crown ethers and cyclodextrins. They are a class of cyclic oligomers prepared from formaldehyde and para-substituted phenols via cyclic condensation under alkaline conditions. Considering the outstanding capacity of the calixarenes as receptors based on their variable chemical modification potential and their conformational pliability, a kind of induced fit to the shape of suitable guest molecules is allowed.

\subsubsection{Chemical Bonding Between Substrates and Sorbent During Sol-Gel Process}

One of the main advantages of the sol-gel process is the presence of chemical bonds between substrate and sorbent. The chemical bond can overcome the mentioned problems related to the conventional sorbents such as low operating temperature and swelling in organic solvents. In doing so, the substrate should be already treated and functionalized. There are three well-known methods to functionalize substrates.

\subsection{Treatment of Fused Silica by $\mathrm{NaOH}$}

Prior to the coating of the SPME fiber by sol-gel technique, the fused silica, as a substrate, is dipped into $1 \mathrm{~mol} \mathrm{~L}^{-1} \mathrm{NaOH}$ solution for $1 \mathrm{~h}$ to activate the surface of the fiber while producing silanol groups, and is then washed with water. The fiber is placed into $0.1 \mathrm{~mol} \mathrm{~L}^{-1} \mathrm{HCl}$ solution for $30 \mathrm{~min}$ to neutralize the excess of $\mathrm{NaOH}$, then washed again with water and dried in the oven. Finally, it is inserted into the prepared sol, and during the sol-gel process and preparation of gel, chemical bonds between sorbent and fused silica substrate are formed (Chong et al., 1997).

\subsection{Self Assembled Monolayers}

The main problem associated with fused silica-based sorbents is the fragility of the fibers. To overcome the mechanical instability of the fibers, metal substrates can be used. As already mentioned, functionalization of the substrate is necessary for sol-gel processing. To prepare a sorbent by sol-gel technology, the substrate must have some functional groups on its surface in order for the chemical bonding with the sorbent to occur. Metal substrates, due to the absence of functional groups, have not been assigned as suitable probes to hold the appropriate sorbents. As there is no functional group on the surface of metals, they have to be functionalized prior to sol-gel process. Self assembled monolayers (SAMs) is a technique which can be easily used to functionalize copper, gold, silver and platinum.

Self-assembled monolayers (SAMs) are ordered monomolecular films which are spontaneously formed by immersing a solid substrate into a solution containing amphifunctional molecules. The amphifunctional molecule has a head group, which usually has a high affinity towards the solid surface, a tail (typically an alkyl chain) and a terminal group that can be used to control the surface properties of the resulting monolayer. The molecular forces between the tails are chiefly responsible for the order of the monolayer. Thus, SAMs have two key features of self-assembly found in biologi- 


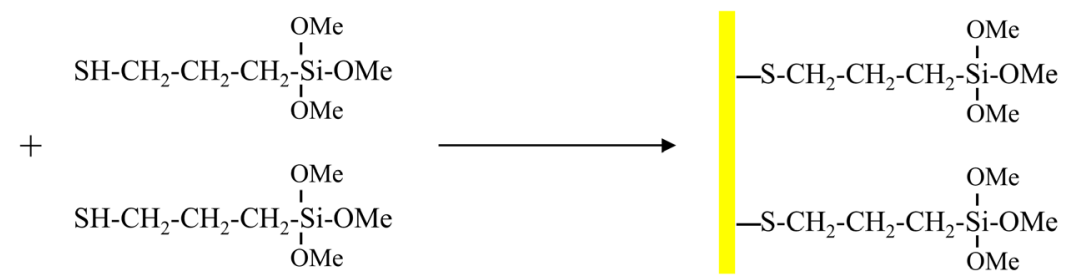

Gold or copper

substrate

Figure 2.9 Schematic diagram of SAMs.

cal systems, namely that molecules have high affinity for each other and predictable structures are formed when the molecular units are associated. The ability to tailor both head groups and tail groups of the self assembling molecules makes control over the self-assembly behavior possible. An alkanethiol has a thiol (-SH) head group and a tail that is usually an alkyl chain. At the end of the tail is the terminal group which, in a well-packed monolayer, determines the properties of the surface of the monolayer. Alkanethiols and di-n-alkyl disulfide self-assemble on coinage metals to form well-organized monolayers, where the formation of a bond between the thiolate head group and the metal surface anchors the organosulfur molecules to the surface and interactions between the alkyl chains give the monolayer its order.

The first application of SAMs to functionalize gold fiber and copper tube was reported by Bagheri et al. (2011b, 2011c, 2011d). Figure 2.9 shows the schematic process involved in SAMs.

Typically, alkanethiols are assembled onto gold surfaces from dilute solutions (millimolar concentrations). Common solvents are ethanol for shorter alkanethiols or hexane for longer alkanethiols. Two distinct adsorption stages are observed in the assembly -a rapid stage within the first few minutes during which time the contact angle is close to its limiting value and the thickness is $80-90 \%$ of the maximum (Bain et al., 1989). The length of this stage is dependent on the alkanethiol concentration, taking only a few minutes at a concentration of $1 \mathrm{mM}$ but about $100 \mathrm{~min}$ at $1 \mu \mathrm{M}$ (Pan, Durning, \& Turro, 1996; Schessler, Karpovich, \& Blanchard, 1996). The second slow stage occurs over several hours as the contact angle and thickness reach their final limits (Bain et al., 1989). During the latter stage, the molecules in the SAM undergo a slow reorganization equivalent to surface crystallization (Karpovich et al., 1998).

\subsection{Diazonium Salts}

Although there has been some extensive progress, SAMs are only amenable to a few metals such as $\mathrm{Cu}, \mathrm{Ag}, \mathrm{Au}$ and Pt. To overcome this limitation, aryl diazonium salts have been used to functionalize several types of metal and non-metal surfaces (Bagheri et al., 2013b). Aryl diazonium salts constitute an important series of compounds in organic chemistry. This is due to the fact that the diazonium group activates 
nucleophilic aromatic substitutions and provides a general pathway for introducing halogens, $\mathrm{CN}, \mathrm{OH}$ and $\mathrm{H}$ into an aromatic ring to form a wide range of compounds. Carbon, metallic, semi-conductor and reduced polymer surfaces can be modified by the electrochemical reduction of aryl diazonium salts with the general formulae $\mathrm{A}^{-},{ }^{+} \mathrm{N}_{2}-\mathrm{C}_{6} \mathrm{H}_{4}-\mathrm{R}$, where $\mathrm{A}^{-}$is the anion and $\mathrm{R}$ stands for different functional groups (Mahouche-Chergui et al., 2011). The interest in using aryl diazonium salts lies in their ease of preparation, rapid (electrochemical) reduction, large choice of reactive functional groups, and strong aryl-surface covalent bonding (Yu et al., 2002; Boukerma et al., 2003). Aryl diazonium salts are excellent alternatives to the traditional silanes and thiols as the former bind polymers mostly to ceramics whereas the latter are mainly limited to the surface of gold, copper and platinum (Love et al., 2005).

To prepare an unbreakable sol-gel CME device using aryl diazonium salts, different metallic substrates such as copper, brass and stainless steel tubes $(0.7 \mathrm{~mm}$ internal diameter) as well as copper, ferronickel, stainless steel wires can be functionalized. Polytetrafluoroethylene (PTFE) can also be used as a non-metal substrate. It is worth noting that the latter must be accomplished using chemical reduction. In all experiments, the capillary tube was used as the HPLC sample introduction loop. Firstly, each capillary tube was washed with acetone (three times), under sonication for $10 \mathrm{~min}$, to clean its internal surfaces. The next step included functionalizing the substrate which involved the electrochemical reduction of 4-aminophenylacetic acid for in situ generation of the aryl diazonium cation (Breton \& Bélanger, 2008). 4-aminophenylacetic acid ( $2 \mathrm{mM})$ was solubilized in aqueous $\mathrm{HCl}(0.5 \mathrm{M})$, and then sodium nitrite ( $2 \mathrm{mM}$ ) was added to solution under magnetic stirring at $500 \mathrm{rpm}$. The mixture was placed at room temperature for about $5 \mathrm{~min}$ to permit completion of the reaction, prior to the electrochemical functionalizing process. The substrates were then inserted in the electrolyte solution while acting as the cathode and a piece of aluminum foil was used as the anode. A peristaltic pump was used to deliver the electrolyte solution through the tube (wires only immersed) while a potential of $-1.5 \mathrm{~V}$ was applied for $15 \mathrm{~min}$. Figure $\mathbf{2 . 1 0}$ shows the electroreduction of the aryl diazonium cation on the inner surface of the copper tubing. To functionalize the surface of PTFE, a representative non-metallic substrate, the PTFE was immersed into the solution of aryl diazonium cation and $150 \mathrm{mg}$ of iron powder was then added. Instantaneously, small bubbles were observed in the flask corresponding to dihydrogen and dinitrogen evolution, as iron reduced protons and diazonium salts in solution (Mévellec et al., 2007). Figure 2.10 shows the general process (Bagheri et al., 2013b).

\subsubsection{Conductive Polymers}

Conductive polymers (CPs) are classified as materials with a highly $\pi$-conjugated polymeric chain (MacDiarmid, 2001; Shirakawa, 2001), exhibiting the electronic properties of both metals and semiconductors and with the mechanical properties of organic 


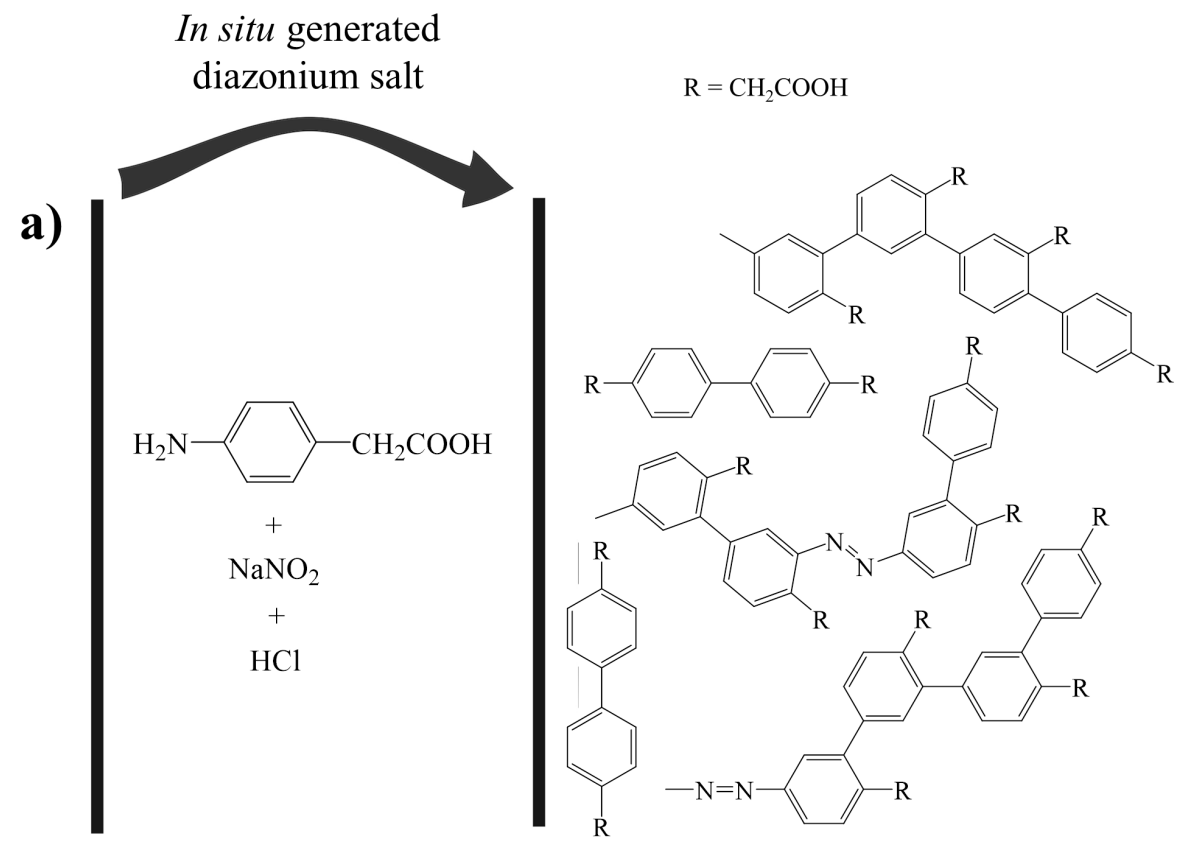

Metal or non-metal substrate

b)

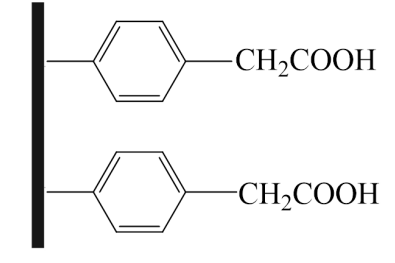

Metal or non-metal substrate

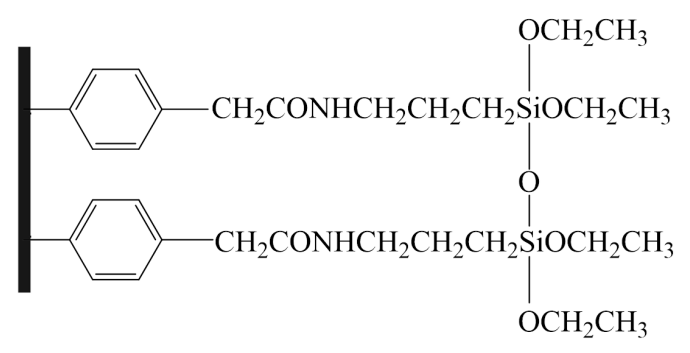

Figure 2.10 (A) Functionalization of any metal or non-metal substrates prior to the treatment and after treatment with aryl diazonium; and (B) coating of the sol-gel sorbent on the inner surface of the functionalized HPLC loop. Reprinted from Bagheri et al. (2013b) with permission from Elsevier. 


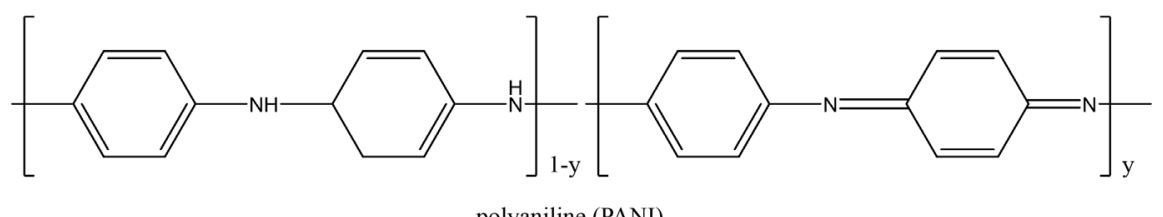

polyaniline (PANI)

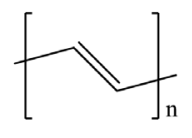

polyacetylene (PAc)

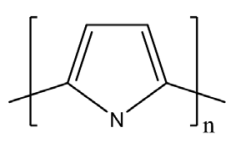

polypyrrole (PPy)

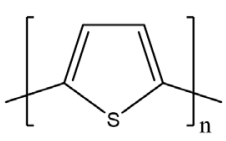

polythiophene (PT)
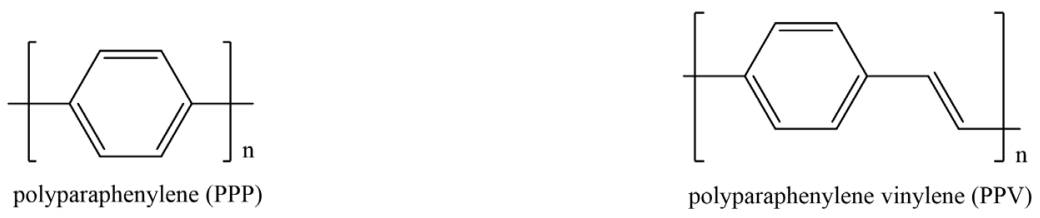

Figure 2.11 Structure of some common conductive polymers.

macromolecules. Research interest in electroactive polymers started in 1977 by preparation of the highly conducting polyacetylene (PAc) and eventually, Heeger, MacDiarmid and Shirakawa were awarded the Nobel Prize in chemistry in 2000 for the discovery and development of the so-called conducting polymers. The conductivity of PAc was demonstrated to be enhanced by several orders of magnitude upon partial oxidation or reduction, which commonly referred to as doping (Shirakawa et al., 1977). CPs have attracted more and more attention due to their electrical properties which are similar to metals but have some characteristics of organic polymers. Furthermore, they can be prepared in such a way as to be applied in different fields. This could be achieved through modifications in the polymer structure and varying the functional groups in the organic moiety. CPs with different chemical structures such as polyene-type, aromatic, heteroaromatic and mixed aromatic (or heteroaromatic) systems (Pron \& Rannou, 2002), polyaniline (PANI), polypyrrole (PPy), polythiophene (PTh), poly(p-phenylene), poly(phenylene vinylene) have been studied extensively. Representative candidates of conducting polymers are given in Figure 2.11. Organic CPS are conformationally rigid molecules due to their extensive conjugation. Most of them are insoluble in common organic solvents while PANI displays a limited solubility in $m$-cresol, 2-pyrrolidinone and concentrated sulfuric acid, whereas no report on PPy solubility can be found (Bagheri et al., 2005b). Additionally, these polymers tend to be infusible and they are usually brittle and have poor mechanical properties. All of these properties are probably related to energetically favorable interactions of the 
$\pi$-systems of closely-linked chains which are stronger than those of polymer with solvent, which probably explain the insolubility and the infusibility of these polymers. Moreover, their mechanical properties derive from the anisotropic character of the intermolecular $\pi$-system interactions producing an amorphous bulk material. Nevertheless, PANI and PPy are of high interest due to their interesting properties such as tunable conductivity and stability in air (Lu et al., 2006). Due to their multifunctionality, ease of synthesis and stability, CPs are attractive and efficient sorbents for the sample preparation purposes. Among sorptive-based extraction methodologies, SPME has been used extensively in the purification and preconcentration of a vast variety of analytes and chemical species from environmental and biological samples. The microextraction techniques rely on quantitative but non-exhaustive transfer of analytes by equilibration of small portions of adsorbents or sorbents and larger amounts of samples, which are in direct or indirect contact (headspace) with each other.

The retention of target analytes in these sorptive-based techniques is due to reversible hydrophobic, polar and ionic interactions between the analytes of interest and the sorptive material. The type of coating used in SPME is therefore responsible for the efficiency of the extraction process. The availability of different materials is one of the advantages of the sorptive-based techniques. The use of CPs as efficient sorbents for sample preparation purposes is mostly due to their multifunctionality and the ability to control their structure so as to influence adsorption processes.

\subsubsection{Structures of Some Well-known Conductive Polymers}

Generally, it is assumed that CP conductivity is related to the higher degree of crystallinity and better alignment of the chains. However, this could not be confirmed for PANI, which is largely amorphous. The basic chemical backbones of conducting PAc, PPy and PANI are shown in Figure 2.12. PANI has different structures considering the level of reduced (amine, NH) and oxidized state (imine, N) of nitrogen atoms in its structure. Concerning emeraldine, half of the total nitrogen along the chain is in the reduced state and the remaining in the oxidized state. As a consequence, the configuration of the neighboring rings is either in a benzenic-like or quinoid-like structure, respectively.

Many other structures are known where nitrogen is in the completely reduced state (leucoemeraldine) or in the fully oxidized state (pernigraniline). Between these two limits, a very large number of intermediate states could exist. However, only a few of them, such as protoemeraldine and nigraniline, are rather stable (Figure 2.12C) (Snauwaert et al., 1990). The emeraldine form of PANI contains an equal number of imine and amine nitrogen atoms, the former being more basic and thus undergoing an easier protonation compared with the amine sites, which are protonated only under highly acidic conditions (Blinova et al., 2006). The secondary amine group (-NH-) in pyrrole is even less basic than the corresponding group in aliphatic compounds. 
a)<smiles>C=C/C=C/C=C/C=C/C=C/C=C/C=C/C=C/C=C/C=C/C=C/C=C/C=C/C=C/C=C/C=C/C=C/C=C/C=C</smiles>

U)<smiles>CC(C)(C)c1ccc(-c2ccc(-c3ccc(-c4ccc(C(C)(C)C)[nH]4)[nH]3)[nH]2)[nH]1</smiles>

C)

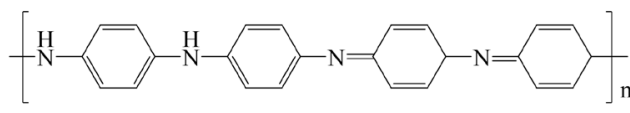

Emeraldine

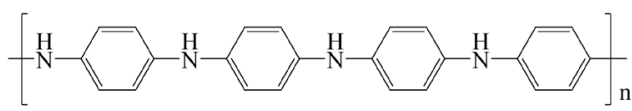

Leucoemeraldine<smiles>CN=C1C=CC(N=C2C=CC(N=C3C=CC(N=C4C=CC(CC(C)(C)c5ccccc5)C=C4)C=C3)C=C2)C=C1</smiles>

Pernigraniline
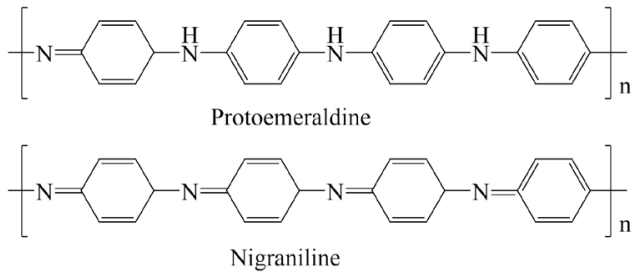

Figure 2.12 (A) Mechanism of electroconductivity in PAc, (B) Chemical structure of PPy, (C) Different forms of PANI.

The acid-base transition in the polymers is responsible for the strong change in their conductivities.

\subsubsection{Preparation of Conductive Polymers}

Organic CPs can be prepared by pyrolysis and numerous methods such as chemical, electrochemical, photochemical, concentrated emulsion, inclusion, solid-state, and plasma polymerization. Among these preparation methods, the electrochemical and chemical polymerizations have been widely applied to the preparation of CPs in industry and other fields of research. Chemical polymerization (Chen \& Tsai, 1993; Wei et al., 1993) is the most useful method for preparing large quantities of CPs, since it is performed without electrodes (Ohtani \& Shimadzu, 1989) but the introduction of reactants and by-products may affect the properties of the produced CPs. Electro- 
chemical polymerization is desirable for conductive polymer thin film and nanostructure fabrication and is normally carried out in a single- or dual-compartment cell by adopting a standard three-electrode configuration in a supporting electrolyte, both dissolved in an appropriate solvent.

\subsection{Chemical Synthesis}

Chemical synthesis is the oldest and the most popular route for the preparation of bulk quantities of CPs on a batch scale. Chemical polymerization is typically carried out using relatively strong chemical oxidants such as $\mathrm{KIO}_{3}, \mathrm{KMnO}_{4}, \mathrm{FeCl}_{3}, \mathrm{~K}_{2} \mathrm{CrO}_{4}$, $\mathrm{KBrO}_{3}, \mathrm{KClO}_{3},\left(\mathrm{NH}_{4}\right)_{2} \mathrm{~S}_{2} \mathrm{O}_{8}$ (Chen \& Tsai, 1993; Wei et al., 1993). These oxidants are able to oxidize the monomers in solution, leading to the formation of cation radicals. These radicals further react with other monomers or n-mers, yielding oligomers or insoluble polymers. Concerning the chemical oxidation of aniline and pyrrole, there are no detailed mechanisms as those reported for the electrochemical polymerization. It is generally accepted that the first step of the polymerization involves the formation of the dimeric species $\mathrm{N}$-4-aminophenylaniline, trans-azobenzene and benzidine. By adapting the chemical procedures, the structure, chemical and physical properties of the obtained polymers heavily depend on the properties of the oxidizing agent, the molar ratio of oxidant to monomer, the electrolyte and the temperature (Ghosh, 2006). Chemical oxidative polymerization is limited by the range of available chemical oxidants. The counterion of the oxidant ultimately will act as a dopant or co-dopant in the polymer. Therefore, it may be difficult to obtain CPs with different dopants. The limited range of oxidants also makes it difficult to control the oxidizing power in the reaction mixture and subsequently the degree of overoxidation during synthesis. Both the type of dopant and the level of doping are influential parameters affecting the final properties of CPs such as molecular weight, crosslinking and conductivity (Freund \& Deore, 2007). Moreover, a strong drawback in using these stoichiometric oxidants is the formation of a large amount of by-products, in the case of ammonium persulfate (APS), the amount of resulting ammonium sulfate is about $1 \mathrm{~kg}$ per $\mathrm{kg}$ of organic polymer.

\subsection{Electrochemical Synthesis}

The electrochemical technique has received wider attention, because of the simplicity and the added advantage of obtaining simultaneously doped CPs. Additionally, in the electrochemical polymerization process a wider choice of cations and anions as dopant ions is available. The electrochemical method also permits the control of film thickness and morphology. In general, chemical oxidation method produces CPs as powders while electrochemical synthesis generally leads to deposition of conducting polymers films onto a supporting electrode surface by anodic oxidation (electropolymerization) of the corresponding monomer (Sadki et al., 2000). When a positive potential is applied to the working electrode, the oxidation starts with the formation of the radical cation. The delocalized radical cations induce the radical-radical 
Monomer oxidation

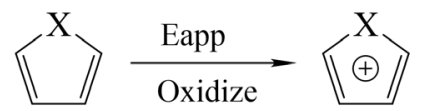

Resonance form

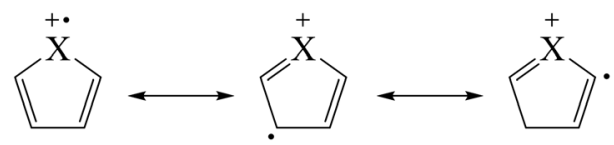

Free-radical coupling

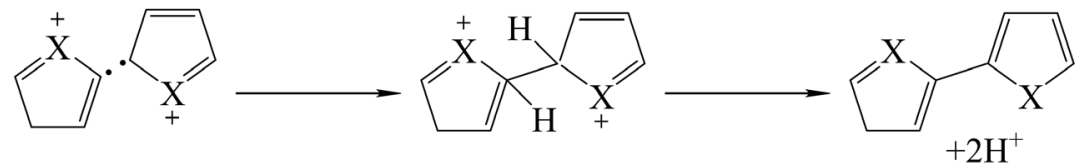

Chain propagation

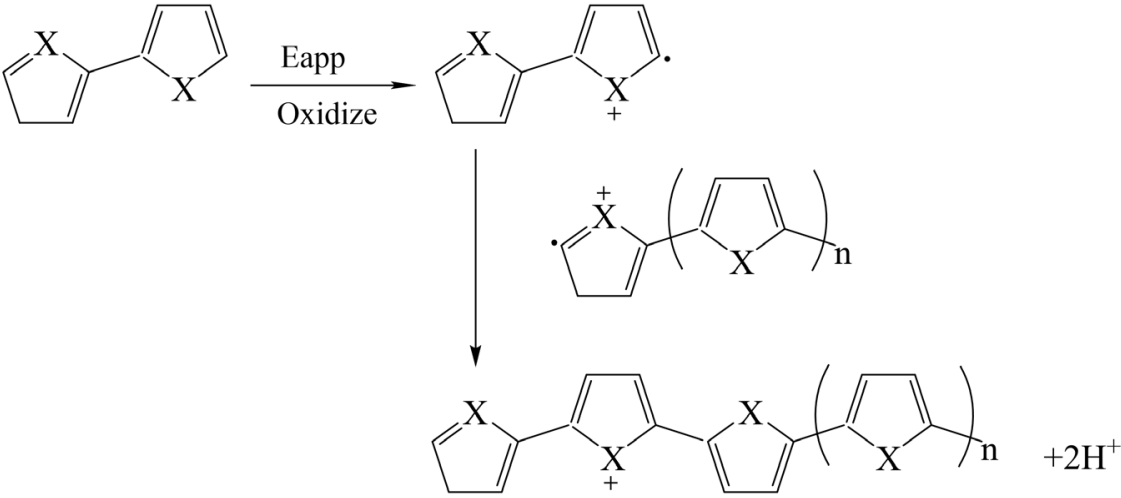

Figure 2.13 Synthesis mechanism of PPy by electrochemical route.

coupling to form dimers by the deprotonation at $\pi$-position. The extended conjugation in the polymer results in a lowering of the oxidation potential compared to the monomer. The electrochemical oxidation and radical coupling process is repeated continuously and finally the deposited CP film is produced on the working electrode. The doping of the polymer is generally performed simultaneously by incorporation of the doping anion into the polymer to ensure the electrical neutrality of the film. The mechanism of electrochemical polymerization of PPy is depicted in Figure 2.13, although the electropolymerization mechanism is rather unknown.

The electrochemical synthesis method has some advantages over the chemical synthesis: (i) a highly electrochemically conductive polymer film can be easily produced on an electrode surface, which is of special interest for applications such as 
modified electrodes, batteries or protective coatings; (ii) film thickness, morphology and conductivity can be easily controlled by monitoring the applied potential and current; (iii) they are cleaner than chemical methods because no oxidizing agent is used; (iv) they provide an in situ condition to investigate the polymerization process and the properties of the resulting conducting polymer.

\subsubsection{Conductive Polymer-based Extractant Phases}

Despite the high specific surface areas of the commercially available hydrophobic sorbents, they suffer from poor interaction and retention of polar compounds which is mostly due to their hydrophobic nature. One solution to this problem is to introduce a polar moiety into their networks to favor the polar interaction between the sorbent and analytes, and enhance the extraction recoveries (Fontanals et al., 2007). Combination of (i) reversed phase mechanism, rising from the polymeric skeleton and (ii) $\pi-\pi$ interactions induced by the available functional groups, provides higher mass transfer which increases the interaction possibilities between the available sorbent sites and the analytes functional groups. All these lead to the enhanced retention of polar compounds. A hydrophilic sorbent can be prepared by copolymerizing monomers containing suitable functional groups or by introducing a functional group to the existing hydrophobic polymers.

\subsection{Polypyrrole-based Coatings}

Tailored properties of PPy coatings can be achieved by proper selection of the counter ion. This dependence has been studied for the determination of volatile organic compounds (VOCs) in headspace mode and for polar and ionic compounds in aqueous solution (Wu et al., 2000). It was reported that the thermal stability and mechanical properties of PPy films could be improved when large aromatic counter anions were incorporated into the films (Nalwa, 1997; Sadki et al., 2000). It was also found that the adhesion of the PPy film to a platinum wire surface was affected by the counter ion used. A more stable PPy coating was produced using polystyrenesulfonate (PSS) as the counterion compared with a similar film containing a chloride counterion. In other work, PPy was synthesized in the presence of different dopants such as chloride, perchlorate, acetate, sulfate, and dodecylsulfate (DS) ions in aqueous medium. Polymer films doped with chloride, perchlorate, and acetate anions showed weak adhesion on the platinum wire and were unstable at temperatures higher than $200^{\circ} \mathrm{C}$. Corresponding thermogravimetric curves revealed that the fibers coated with sulfatedoped PPy (PPy-S) and PPy-DS under argon atmosphere are thermally stable up to $300{ }^{\circ} \mathrm{C}$. In addition, the perfect adhesion of these films to Pt wires was also achieved. The extraction properties of these two fibers toward some PAHs were examined using a HS-SPME set-up. The results showed that PPy-DS possesses up to three times higher extraction efficiency toward the PAHs compared to the PPy doped with sulfate. These results can be explained by the presence of a $C_{12}$ chain alkyl group in the DS ion 
which increased the hydrophobic interaction of PPy-DS film with PAHs (Mohammadi et al., 2005). A PPy modified with tetrasulfonated nickel phthalocyanine (NiPcTS) doping anion was prepared and used for the extraction of BTEX compounds from water samples by headspace solid-phase microextraction (HS-SPME). It was shown that the PPy doped with NiPcTS could extract up to five times as much of the BTEX compounds in comparison with the PPy doped with other anions such as toluene$p$-sulfonate (TOS), tetrafluoroborate $\left(\mathrm{BF}_{4}^{-}\right)$and perchlorate $\left(\mathrm{ClO}_{4}^{-}\right)$. This is probably due to the presence of phenyl groups in NiPcTS incorporated into PPy as counterions (Djozan et al., 2004). The effect of different types of doping ions in PPy-based SPME fibers have been examined for sodium polyphosphate (PP), a new dopant for the electropolymerization of pyrrole on the surface of a steel fiber with the aim of achieving a polymer coating with improved qualities for SPME analysis. It was revealed that the $\mathrm{PPy} / \mathrm{PP}$ coating has the highest extraction efficiency compared with the PPy polymer containing other counter-ions (perchlorate, tartarate and oxalate). In addition, thermogravimetric analysis of $\mathrm{PPy} / \mathrm{PP}$ revealed that it is more thermally stable than other $\mathrm{CPs}$. Also, the prepared PPy/PP coating was physically and thermally more stable and did not show any major weight loss even at $450^{\circ} \mathrm{C}$. Moreover, the PPy doped with PP could overcome the adhesion problem through incorporation of the inorganic PP into the organic polymeric structure, which led to a chemically adhered coating through Fe-P bonding to the metal surface (Rout et al., 2006). Additionally, an enhancement in the extraction efficiency of organochlorines was observed by increasing the PP concentration up to $10 \%$, and then the extraction efficiency reached a plateau region (Mollahosseini \& Noroozian, 2009). Interestingly, the successful application of PPy in SPME fiber coatings as both an extraction phase and a surface to enhance laser desorption/ionization (SELDI) of analytes was reported. The developed SPME/SELDI fiber integrated sample preparation and sample introduction on the tip of a coated optical fiber, which also acted as the transmission medium for the UV laser light. A SELDI surface could be used to transfer the laser energy to ionize the analytes instead of using a matrix (Wang et al., 2004).

\subsection{Polyaniline-based Coatings}

Along with PPy, PANI has been applied as a SPME fiber coating as well. Cyclic voltammetry (CV) has been used to deposit PANI on platinum wire for the determination of phenolic compounds (Bagheri et al., 2005b) and PAHs in water (Bagheri et al., 2007). This polymer exists in a variety of protonated and oxidized forms which can be specifically achieved by adjusting the scan rate and sweep potential (Ghassempour et al., 2005) during the electrocoating process using CV. The leucoemeraldine base form of PANI fiber coating in a setup with gas chromatography-mass spectrometry (GC-MS) provided the highest sensitivity for the determination of anatoxin-a, an alkaloid produced by cyanobacteria from cultured media and contaminated water. Also, the same coating could be prepared on steel (Minjia et al., 2004) and gold (Djozan \& Bahar, 2004) supports by the application of a constant voltage. In order to improve 
the application of CPs as SPME fibers, a trifluoroacetic acid-doped PANI fiber was introduced for SPME (Wang et al., 2009). The extraction capacity of this coating was much greater than commonly used PANI (sulfuric acid-doped PANI) and PTh-based SPME fibers, and comparable to a commercially available $30 \mu \mathrm{m}$ PDMS fiber. This SPME fiber coating had a thinner stationary phase but proved to have high thermal stability. Fluorine in this fiber was thought to play an important role in the explanation of its good performance. To further increase the extraction efficiency and to test the role of fluorine, a perfluorinated acid has been introduced to the PANI backbone and applied to the SPME fiber. It was reported that the perfluorooctanesulfonic acid-doped PANI (PFOS-PANI) was superhydrophobic. It was demonstrated that the PFOS-PANI coating, roughly $10 \mu \mathrm{m}$ thick, exhibited high extraction efficiency for polybrominated diphenyl ethers (PBDEs), phenols, polychlorinated biphenyls (PCBs) and PAHs in comparison with the $100 \mu \mathrm{m}$ PDMS, $85 \mu \mathrm{m}$ PA and $65 \mu \mathrm{m}$ PDMS/DVB fibers (Wang et al., 2010). In addition, the prepared PANI fiber coating showed to have high thermal (up to $350{ }^{\circ} \mathrm{C}$ ) and solvent stability compared to the commercial fibers (Li et al., 2007). In other work, a poly(p-phenylenediamine-co-aniline) composite SPME coating was prepared on the surface of a stainless steel wire via electrochemical deposition (Rong et al., 2012). Interestingly, adapting a novel deposition approach, SAMs of polyaminothiophenol (PATP) were used as a covalently bonded coating for SPME. Thiolated aniline-analog monomers (mixture of 2- and 3-aminothiophenols, 2/3-ATP) were anchored on the gold surface and then electropolymerized. The thiolterminated coating on the gold surface was very stable due to the strong S-Au bond. The proposed covalently bonded coating showed higher mechanical (re-usability up to 100 times) and thermal stability (up to $320^{\circ} \mathrm{C}$ ) than a non-covalent bonded PANI coating which could be reused up to 20 times and its thermal stability was just below $250^{\circ} \mathrm{C}$ (Mehdinia et al., 2011).

\subsection{Polythiophene-based Coatings}

PTh was firstly proposed as SPME fiber by Li et al. (2008). Thiophene is a heterocyclic aromatic monomer and due to its high oxidation potential is rather difficult to be electropolymerized (Gratzl et al., 1990). In order to resolve this issue, a solution containing boron trifluoride diethyl etherate (BFEE) was used, which could act as a strong Lewis acid while possessing good ionic conductivity and electrocatalytic properties. The complexation of aromatic monomers of thiophene with BFEE can significantly lower the anodic oxidation potentials of the monomers, which is beneficial to the preparation of high quality CPs (Shi et al., 1995). Thus, the electropolymerization of thiophene can be carried out in pure BFEE. There are limited reports in the field of application of polythiophene as a SPME coating. Olszowy et al. have applied PTh and PPy as sorbent phases for SPME in order to extract multi-resistant antibiotic drugs such as linezolid and daptomycin from whole blood sample followed by HPLC-UV detection. The PTh SPME coating was shown to have better adsorption capacity compared to a PPy coating for both drugs (Olszowy et al., 2010). In other work, the PPy 
and PTh coatings were electropolymerized to be used in SPME analysis after which the fibers were modified by an ozone treatment in a gaseous phase. Extraction efficiencies of both kinds of fibers were compared by the microextraction of linezolid from standard solutions. In these investigations a better adsorption capacity was obtained for PPy fibers (Olszowy et al., 2011).

\subsubsection{Monolithic Polymers}

\subsubsection{Preparation of Monolithic Polymers}

Organic monolithic polymers are porous materials that can be synthesized in a onestep polymerization procedure. The pre-polymerization mixture consists of the monomers, cross-linkers, porogenic solvents, and initiators. Figs. 2.14 and 2.15 show some compounds usually employed as monomers and cross-linkers, respectively. A container is filled with the pre-polymerization mixture and the polymer can be synthesized via a free-radical mechanism. The prepared polymer forms the container shape by in situ polymerization (Bagheri et al., 2013c). Polymerization can be initiated by heating, UV radiation or gamma-ray radiation in the presence of initiators. After reaction for the prescribed time at an appropriate temperature, the resulting material is washed with organic solvents to remove the unreacted components and porogenic solvents. Monolithic polymers are highly porous materials with a network of interconnected pores and have been utilized in many applications in separation science such as chromatography stationary phases and also as the extracting phase in solid phase extraction (SPE) and SPME. They are generally highly biocompatible and pH-stable, making them suitable for biological samples (Svec, 2010). However, in most cases, they suffer from shrinkage or swelling when exposed to organic solvents, leading to poor mechanical stability.

To obtain a large surface area, a large number of small pores should be incorporated into the polymeric network. A highly substantial contribution to the overall surface area originates from the micropores, with sizes smaller than $2 \mathrm{~nm}$, followed by the mesopores which range from 2 to $50 \mathrm{~nm}$ (Svec, 2010). Influencing variables such as temperature, composition of the porogenic solvent and content of cross-linker have major roles in the tuning of the pore size over a broad range.

Temperature: The polymerization temperature, via its effects on the kinetics of polymerization, is particularly effective in controlling pore size distributions. The effect of temperature can be explained in terms of the nucleation rates and the shift in pore size distribution induced by changes in temperature (Svec \& Frechet, 1995; Svec, 2010). The temperature mostly affects the specific surface area which is typically related to the small pores. Since the monomers are better solvating agents thermodynamically than the porogenic solvent, the precipitated insoluble gel-like nuclei swell with the available monomers in the surrounding liquid. Following the nucleation step, the polymerization continues both within the separated phase of monomer 


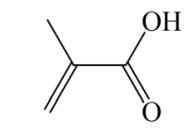

methacrylic acid

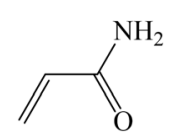

acrylamide

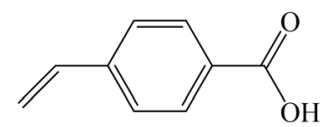

p-vinylbenzoic acid

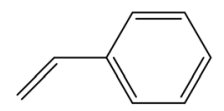

styrene

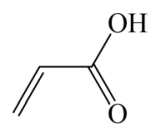

acrylic acid<smiles>C=C(C)C(=O)OCCCC</smiles>

butyl methacrylate

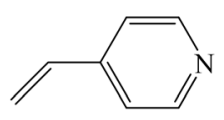

4-vinylpyridine<smiles>C=C(C)C(=O)OC</smiles>

methyl methacrylate<smiles>C=C(C)C(=O)OCCCCCCCCCCCC</smiles>

Figure 2.14 Some monomers used for monolithic polymer preparation.

swollen nuclei and in the remaining liquid polymerization mixture. At low temperatures, the reaction rate is rather slow and transfer of a substantial part of monomers from solution to the nuclei can occur. Polymerization within the nuclei is kinetically preferred because the local concentration of the monomers is higher than in the surrounding solution. By increasing the temperature, the number of polymer molecules that are formed in the solution after the original nucleation increases. These are captured by the growing nuclei and form larger clusters with less individualized texture and a reduction in the surface area.

Cross-linker: Changing the monomer to cross-linker ratio not only induces the formation of different porous structures but also leads to materials with different compositions. A higher content of cross-linker causes the formation of more crosslinked polymers in the early stages of the polymerization process and therefore earlier phase separation can occur. The nuclei are more cross-linked and since this crosslinking has inverse effects on their swelling with the monomers, their sizes remain relatively small. Since the final structure consists of smaller globules, it also has smaller voids. Thus, this approach is useful for the preparation of monoliths with very large surface areas (Santora et al., 2001). Overall, the pore size distribution is controlled by the swelling of cross-linked nuclei.

Porogenic solvent: The choice of porogenic solvent is another option that may be used to control porous properties without changing the chemical composition of the final polymer. In general, larger pores are obtained using a weaker solvent due to an earlier onset of phase separation. The porogenic solvent controls the porous properties of the monolith through the solvation of the polymer chains in the reaction medium during the early stages of the polymerization. The choice of porogenic solvent for the preparation of porous polymer monoliths mostly relies on the exper- 


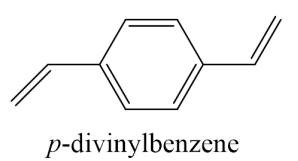

p-divinylbenzene

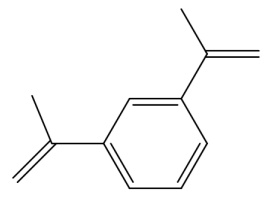

1,3-diisopropenyl benzene<smiles>C=C(C)C(=O)OCCOC(=O)C(=C)C</smiles>

ethylene glycol dimethacrylate<smiles>C=C(C)C(=O)NCCNC(=O)C(=C)C</smiles>

N,N'-ethylene bismethacrylamide

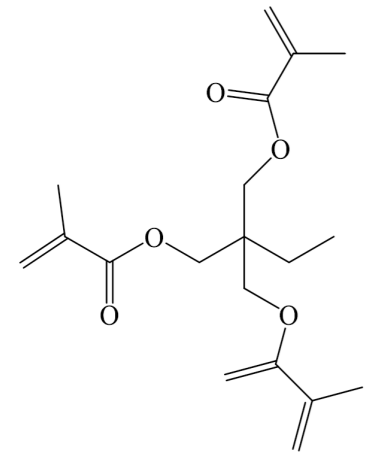

trimethylpropane trimethacrylate

Figure 2.15 Some cross-linkers used for monolithic polymer preparation.

tise of researchers. That might be the reason why few porogenic solvents have been used and most often proven porogen mixtures are applied.

Time: In the polymerization process, relatively low duration times result in low yields for polymer reactions and most of the monomers remain non-polymerized. This initial monolith has a high pore volume and surface area. After a quantitative conversion of monomers to polymer at extended time, the surface area and pore volume are decreased.

\subsubsection{Monoliths for Solid-phase Microextraction}

Monolithic polymers have been used in SPME under two categories: fiber and in-tube formats.

\subsection{Fiber Format}

A monolithic fiber was constructed from methacrylic acid-ethylene glycol dimethacrylate (MAA-EDMA) for selective extraction of diacetylmorphine and analogous compounds (Djozan \& Baheri, 2007). In another work, a monolithic fiber-based MIPSPME was developed for selective and sensitive determination of ephedrine and pseudoephedrine (Deng et al., 2012). Polymerization of MAA, EDMA and ephedrine as a template was performed in situ in a silica capillary mold. In another publication, a MIP-SPME fiber was synthesized for the extraction of acetaldehyde from the headspace of beverages stored in poly(ethylene terephthalate) bottles (Rajabi Khorrami 


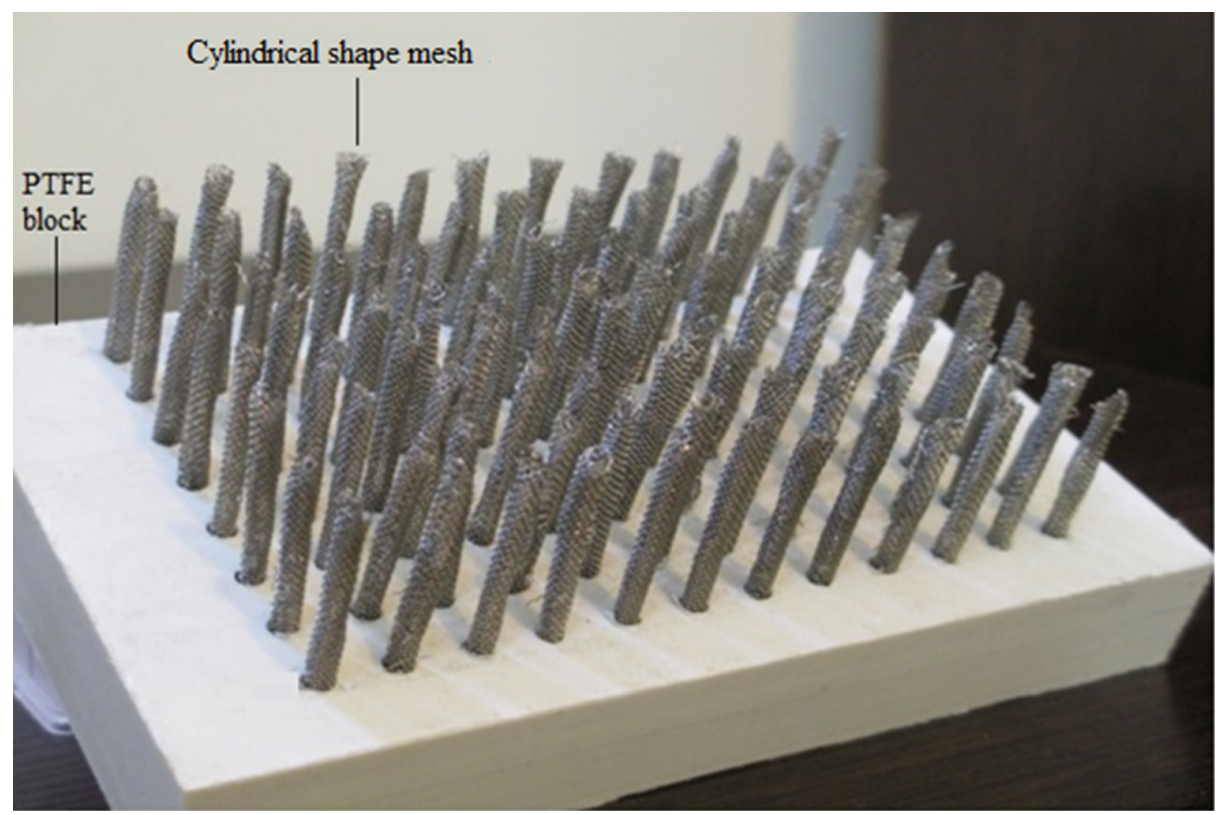

Figure 2.16 Photo describing the homemade 96 well $\mu$-SPE device. Reprinted from Bagheri et al. (2013c) with permission from Elsevier.

\& Narouenezhad, 2011). Djozan et al. reported a simple polymerization strategy to produce a monolithic SPME fiber on the basis of MIP for selective extraction of triazines from water, rice and onion samples (Djozan \& Ebrahimi, 2008).

Recently, a novel high-throughput device based on a 96-micro-solid phase extraction ( $\mu$-SPE) system with monolithic sorbent was reported (Bagheri et al., 2013c). The extraction procedure was performed on a commercially available 96-well plate system. The extraction module consisted of 96 pieces of $1 \times 3 \mathrm{~cm}$ of cylindrically shaped stainless steel meshes. The prepared meshes were fixed in a homemade PTFE-based block with 96 holes for possible simultaneous immersion of meshes into the center of individual wells (Figure 2.16). Dodecyl methacrylate and EDMA were copolymerized as a monolithic polymer with pyramid shape (Figure 2.17). These sorbents were placed in the cylindrically shaped stainless steel meshes. The method was successfully developed for the extraction and determination of the some selected pesticides in water samples.

\subsection{In-tube Solid-phase Microextraction}

Because of fragility, poor mechanical stability of monolithic polymers and low extraction capacity, in-tube format of SPME has gained more attention. This format increases the range of available coatings and conveniently integrates the extraction (on-line or off-line) with separation and detection systems. This setup in particular is quite promising when SPME is coupled with HPLC. 


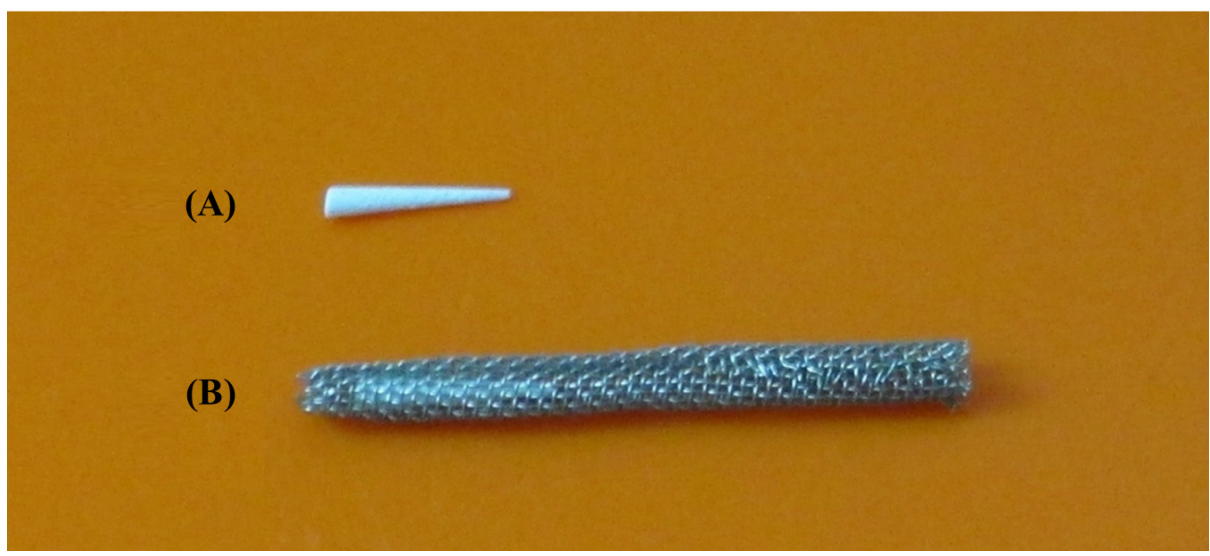

Figure 2.17 (A) A monolithic polymer with pyramid shape, (B) A cylindrical shaped mesh. Reprinted from Bagheri et al. (2013c) with permission from Elsevier.

Monolithic materials are always prepared in situ without the need for frits (Figure 2.18). Due to their satisfactory phase ratios and bimodal nature, monolithic materials result in convective mass transfer rather than diffusive mass transfer during the extraction process, which is a more desirable feature for high speed extraction.

In the early works on organic monolith, Fan et al. (2004) reported the use of a poly MAA-EDMA monolithic capillary as an in-tube microextraction. Because of polymer biocompatibility, only simple sample pre-treatment before extraction was required. Since then, monolithic polymers with different functional groups have been constructed for a variety of SPME applications (Fan et al., 2004; Zhang et al., 2006; Zheng et al., 2009, 2010; Chen et al., 2012; Wang et al., 2013; Zhang \& Chen, 2013).

\subsubsection{Composites}

Composite materials are solids resulting from the combination of two or more simple materials that contain a continuous phase (polymer, metal, ceramic, etc.), and a dispersed phase such as glass fibers, carbon particles, silica powder, clay minerals and other relevant materials. In addition, composite materials have properties that are quite different from the components taken separately. Within the vast collection of inorganic-organic hybrid materials, composites are an emerging group that received a great deal of attention not only because of their potential in industrial applications but also from their scientific point of view (Ahlrichs et al., 1975; Akelah et al., 1994). Important advantages of composites over their constituent compounds rely on their high specific stiffness, strength, toughness, corrosion resistance, low density and thermal insulation. In most composite materials, one phase is usually continuous and called the matrix, while the other phase called the dispersed phase. On the basis of the nature of the matrices, composites can be classified into four major categories: 


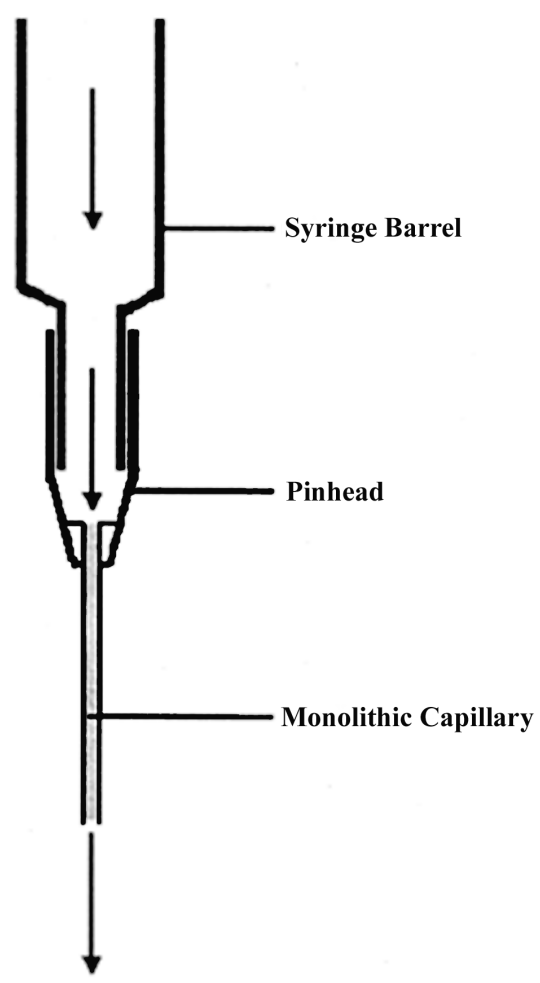

Figure 2.18 Scheme of the in-tube monolith microextraction. Reprinted from Zhang et al. (2006) with permission from Elsevier.

1. Polymer matrix composites

2. Metal matrix composites

3. Ceramic matrix composites

4. Carbon matrix composites.

Polymer matrix composites can be processed at a much lower temperature in compared with other composites. Depending on the types of polymer, polymer matrix composites are classified as thermosetting composites and thermoplastic composites.

\subsubsection{Polymer Matrix Composites}

To improve the properties of composite-based materials, the investigation of composites have been performed with lower filler sizes which led to the development of microcomposites and nanocomposites. Nanocomposites refer to composites in which one phase has nanoscale morphology such as nanoparticles, nanotubes or lamellar nanostructure (Friedrich et al., 2005). The improvement of the properties by the addition of particles can be achieved when (i) adequately good interactions between the 
nanoparticles and the matrix exist and (ii) the doped particles (nanoparticles) are well dispersed within the matrix.

In nanocomposites, covalent bonds, ionic bonds, van der Waals forces and hydrogen bonding interctions may exist between the matrix and filler components. Two types of the most common nanofillers including nanoparticles and nanoclays are under active investigation. The main purpose is to have a valuable nanocomposite with the largest possible surface area of nanofillers. In practice, production aims to avoid aggregation of nanoparticles and exfoliation of nanoclays. Nanoparticles are commercially available from different sources. Sols of nanosilica as colloid solutions in water or in organic solvents are used in the preparation of nanocomposites. Fused silica is available as individual particles ranging from $10-20 \mathrm{~nm}$ to micrometers, and can be more or less successfully dispersed in a polymer (Zhou et al., 2002). Layered aluminosilicate clays and especially montmorillonite (bentonite) are widely used in nanocomposites. Silicates have a characteristic interlayer distance of $1 \mathrm{~nm}$; the basal spacing of a gallery is also ca. $1 \mathrm{~nm}$. Inorganic cations like $\mathrm{Na}^{+}$between galleries hold negatively charged galleries together. The replacement of the inorganic cations in the galleries of the native clay by alkylammonium (onium) salts or quarternary amines with long alkyl substituents (surfactants) leads to a better compatibility between the inorganic clay and hydrophobic polymer matrix. The replacement leads to an increase in the space between galleries which facilitates the intercalation of polymer molecules into the clay.

The preparative methods of nanocomposites are divided into three main groups based on the starting materials and processing techniques. The first group is based on the intercalation of polymer or pre-polymer from solution. Here, a solvent system is chosen in which the polymer or pre-polymer is soluble and the silicate layers can be swollen. The layered silicate is first swollen in a solvent, such as water, chloroform, or toluene. When the polymer and layered silicate solutions are mixed, the polymer chains intercalate and displace the solvent within the interlayer of the silicate. After solvent removal, the intercalated structure remains, leading to the desired nanocomposite. In this method, which is also named the in situ intercalative polymerization method, the layered silicate is swollen within the liquid monomer or a monomer solution so that polymer formation can occur between the intercalated sheets. Polymerization can be initiated either by heat or radiation, by the diffusion of a suitable initiator or by an organic initiator or catalyst fixed through cation exchange inside the interlayer before the swelling step.

The second technique is called the melt intercalation method. This method involves annealing a mixture of the polymer and silicate above the softening point of the polymer. This method has great advantages over either in situ intercalative polymerization or polymer solution intercalation. First, this method is environmentally friendly as no organic solvent is needed. Second, it is compatible with current industrial processes such as extrusion and injection molding. The melt intercalation method allows the use of polymers which were previously not suitable for in situ 
polymerization or solution intercalation. In addition, polymer nanocomposites can commonly be obtained by the sol-gel method. Sol-gel nanocomposites (polymer/ silica nanocomposite) are prepared by in situ hydrolysis and condensation of mononuclear precursors such as tetraethoxysilane (TEOS) and tetramethoxysilane (TMOS) in organic polymer matrices (Lan et al., 1995; Tong et al., 2002). Recently, the intercalation method for synthesizing polymer/clay nanocomposites has received more attention where polymer chains may penetrate into the host layers while ordered silicate registry remains (intercalated structure), or the exfoliated individual silicate layers (about $1 \mathrm{~nm}$ thickness) are homogeneously dispersed in the organic polymer matrix (delaminated structure) (Krishnamoorti et al., 1996). Since the successful synthesis of a nylon 6/clay nanocomposite, many polymer/clay nanocomposites have been reported (Kawasumi et al., 1999). Research indicates that, compared to the intercalated nanocomposites, the exfoliated nanocomposites have higher Young's modulus, a larger increase in elongation at the breaking point and better thermal stability and the extent of exfoliation strongly influences the improvement of the properties. Natural silicates have strong interactions between the layers which are due to negative charges and hydrogen bonding in their structures. The basal space of pristine silicate is about $1 \mathrm{~nm}$, which is smaller than the radius of gyration of general polymers. This might present an obstacle for polymers to penetrate into or delaminate between the silicate layers. Thus, most hydrophobic polymers are limited in terms of penetration depth into layered regions of hydrophilic silicates. So far, the preparation of polymer/silicate nanocomposites has been mostly focused on the use of poly(ethyl acrylate)/bentonite nanocomposites.

\subsubsection{Nanocomposite-based Coatings}

Recently, advancements in nano-oriented science and the synthesis of nanomaterials, nanoparticles and nanocomposites have drawn the attention of researchers in different fields. Nanomaterials, specifically due to their high surface area and more efficient availability of the adsorption sites, have attracted more attention for use as the extracting phase for sample preparation purposes and among them, nanocomposites are quite dominant. In this regard, some reports concerning the preparation and application of polymer-based nanocomposites as SPME fiber coatings have been appeared in the literature. For instance, a novel nano-structured polyanilineionic liquid (1-butyl-3-methylimidazolium hexafluorophosphate, BMIPF ${ }_{6}$ ) composite (BPANI) film coated on steel wire was prepared by electrochemical deposition (Gao et al., 2011). Scanning electron microscopy (SEM) images showed that the obtained porous BPANI coating consisted of nanofibers, with diameters ranged from 50 to 80 $\mathrm{nm}$. The BPANI coating showed better analytical performance toward organochlorine pesticides (OCPs) in compared with conventional PANI and PDMS coatings. Furthermore, the novel nano-structured composite coating was very stable at relatively high temperatures (up to $350^{\circ} \mathrm{C}$ ) and it could be used more than 250 times without 
obvious reduction in the extraction efficiency. It was reported that the polymerization of aniline in the presence of $\mathrm{BMIPF}_{6}$ can enhance the thermal stability of the PANI film (Gao et al., 2004) and the enhanced temperature resistance may be due to the introduction of fluorine into the polymeric network (Mecerreyes et al., 2002). Later on, a novel nano-structured copolymer of aniline and $m$-aminobenzoic acid ( $m$-ABA), with a diameter lower than $75 \mathrm{~nm}$, was introduced as a SPME coating for isolation of saturated fatty acids in zooplanktons (Mehdinia et al., 2010). Improved temperature resistance (up to $350{ }^{\circ} \mathrm{C}$ ), relatively improved lifetime (more than 50 extractions) and satisfactory extraction efficiencies were obtained by incorporating carboxylate groups into the framework of PANI. In another study, the effects of structure and morphology of the SPME coating on the equilibrium extraction time was investigated. PANI was used as an extraction phase for the comparison of extraction capacity and equilibrium extraction time of nano- and micro-structured coatings. PCBs were used as model compounds to examine the extraction properties of nano- and micro-structured coatings. The results revealed that the nano-structured PANI coating, due to its larger surface area, exhibited a higher extraction rate and shorter desorption time in comparison with the micro-structured coating (Mehdinia \& Mousavi, 2008). In another study, pristine and oxidized multi-walled carbon nanotubes-polypyrrole composite (MWCNTs-PPy) deposited electrochemically on a stainless steel wire (Asadollahzadeh et al., 2010; Chen et al., 2011) and MWCNTsPANI film coated platinum wire ( $\mathrm{Du}$ et al., 2009) were used for the extraction of pyrethroids, phthalate esters and phenolic compounds from aqueous samples. It was reported that the electrodeposited coating presents a porous structure with high specific surface area and adsorption capacity and thus higher extraction efficiency for the analytes. This might be due to the fact that both MWCNTs and CPs contribute to the extraction. Moreover, a novel PANI and polyamide nanocomposite based on nanosilica was synthesized and was used as SPME coating. In this study nanosilica was used as a reinforcing agent to improve the extraction capability of PANI. Evaluation of PANI polymer and aniline-silica nanocomposite fibers by SEM has shown that the synthesized nanocomposite coating possesses a non-smooth, porous structure in comparison with the PANI film. In addition, the use of the aniline-silica nanocomposite led to higher extraction results in comparison with a PANI fiber (Bagheri \& Roostaie, 2012). A PPy/graphene (G) composite was prepared and applied as a novel SPME coating for determination of phenols. The PPy/G-coated fiber was synthesized by electropolymerization of pyrrole and $G$ on a stainless-steel wire. The extraction efficiency of PPy/G-coated fiber for phenols was the highest in comparison with either PPy or PPy/graphene oxide (GO) fibers. Extraction capability of a novel $\mathrm{PPy} / \mathrm{G}$-coated fiber was evaluated by comparison with the extraction efficiency of 85 $\mu \mathrm{m}$ CAR/PDMS fiber and a $85 \mathrm{~mm}$ PA fiber and the results showed that the extraction efficiency of PPy/G coated fiber is better or comparable compared to the tested commercial fibers (Zou et al., 2011). 


\subsubsection{Electrospun Nanofibers}

Today, nanomaterials are considered to be a well-established and interesting class of materials due to their unique physical properties and small sizes. These properties could improve and bring new features which are currently difficult to achieve with bulk materials (Gómez-Hens et al., 2008). Recently some research groups tried to prepare nanomaterial-based SPME fibers (Mehdinia et al., 2006, 2011; Cao et al., 2008; Rastkari et al., 2010; Zewe et al., 2010). An essential and rather important characteristic of sorbents is its surface area-to-volume ratio, with materials having long lengths and nano- to microscale diameters possessing high surface area-to-volume ratio (Huang et al., 2003). These nanomaterials can be regarded as good candidates to be used as extracting phases in analytical and environmental applications. Electrospinning is a simple and convenient method for producing nanofibers with adjustable diameters, polarities and porosities. The nanofibers have malleability to conform to a wide variety of sizes and shapes. It is possible to control the nanofiber composition to achieve the desired properties and functionality (Bhardwaj \& Kundu, 2010). A variety of polymers have been used for producing nanofibers (Huang et al., 2003). The electrospun nanofibers have found a great deal of applications in medicine, enzyme immobilization, batteries, fuel cells, capacitors, filter membranes, drug delivery, tissue engineering, sensors and protective cloth for warfare agents (Ramakrishna et al., 2005). This methodology has been used in SPE (Kang et al., 2007; Xu et al., 2010), semi-micro-SPE (Chigome et al., 2010), affinity membranes (Yoshimatsu et al., 2008) and more recently as a SPME fiber (Zewe et al., 2010). The electrospinning process operates based on similar principle as electrospray ionization mass spectrometry (ESI-MS) (Rutledge \& Fridrikh, 2007). In this approach, a fiber jet is produced rather than the charged droplets mist in ESI-MS. In the electrospinning process, a solution of a high molecular weight polymer with high viscosity is used. When the electrostatic repulsion overcomes the surface tension, the spherical droplets deform to Taylor conical form. By increasing the voltage across the polymer-containing syringe and the collector, the jet of the polymer ejects from the Taylor cone. The entanglement of polymer chains and the surface tension prevent the formation of polymeric droplets. As the polymer jet is flying towards the collector electrode, its diameter gets smaller. At the end of the process, a non-woven nanofiber mat is collected on the surface of the conductive collector, possessing nano- to microscale diameters. Miscellaneous materials with different shapes can be used as collector electrodes (Teo \& Ramakrishna, 2006). In this regard, some reports concerning the preparation and application of polyurethane- and polyamide-based nanofibers as SPME fiber coatings have appeared in the literature. For instance, a novel polyamide nanofiber coated on a steel wire was prepared by the electrospinning technique (Bagheri \& Aghakhani, 2011, 2012). SEM images showed that the obtained porous polyamide coating consisted of nanofibers, with diameters ranging from 150 to 600 $\mathrm{nm}$. It was found that the electrospun coating presents a porous structure with high 


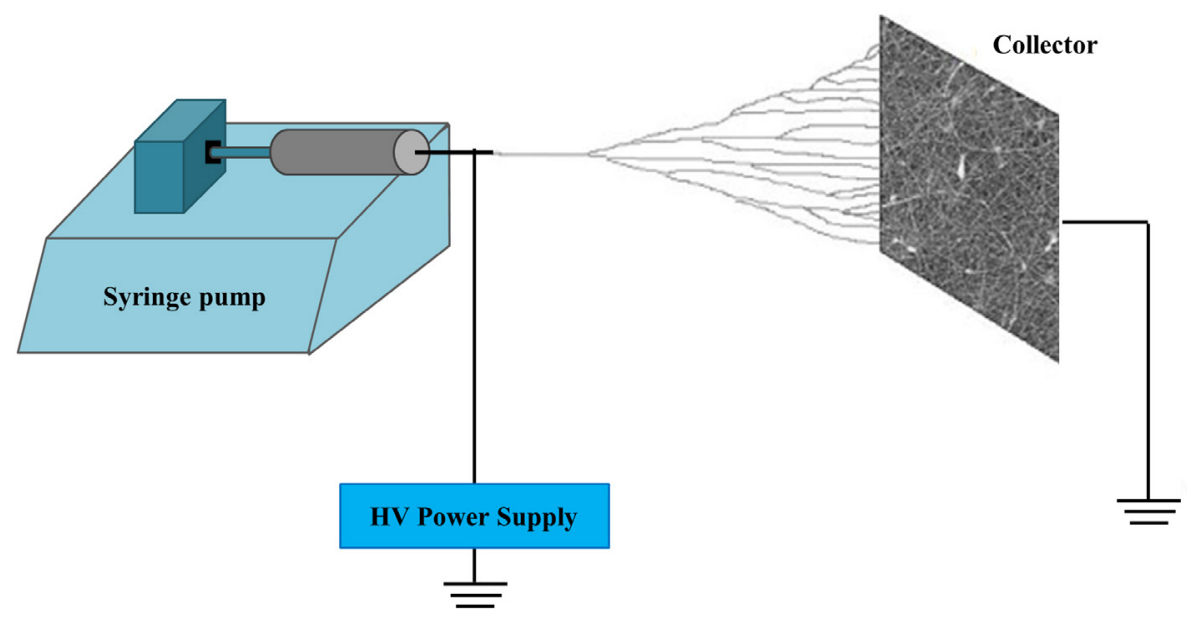

Figure 2.19 Scheme of the electrospinning setup. Reprinted from Bagheri \& Aghakani (2012) with permission from Elsevier.

specific surface area and adsorption capacity, and thus high extraction efficiency for the analytes (Figure 2.19).

\subsubsection{Selective Sorbents}

Preparation of selective sorbents is achievable through MIPs. The principle of molecular imprinting was inspired by Fischer's lock-and-key metaphor. In the first step, the selected key molecule is mixed with lock building blocks. The building blocks and the key are allowed to associate with each other, firmly or loosely. The complexes that are formed between the key and the building blocks are subsequently glued together in order to fix the building block positions around the key. Removing the molecular key then leaves a construction which, if everything works properly, is selective for the original key. In molecular imprinting, molecules are used to create the marks or imprints, normally within a network polymer. In molecular imprinting, the key molecules described above can be denoted by a variety of expressions (e.g., templates, template molecules, target molecules, analytes, imprint molecules, imprint antigens or print molecules), any of which is frequently encountered. The lock building blocks are normally called functional monomers, although polymers have also been used as imprinting building blocks. The molecular glue used to fix the key-building block complexes is almost always perceived as a cross-linker or a cross-linking monomer. 


\subsubsection{Molecularly Imprinted Polymers-based-solid-phase Microextraction}

The combination of MIP and SPME technique provides a powerful sample preparation tool in terms of selectivity, simplicity, and flexibility. The first attempt to use MIPs in CME (in-tube SPME) was reported by Mullett et al. (2001). An automated, online MIP-SPME method was developed for the determination of propranolol in biological fluids and showed improved selectivity compared to in-tube stationary phase materials, thus overcoming the limitations of the existing SPME coating materials. Preconcentration of the sample by the MIP adsorbent increased the sensitivity, yielding low limits of detection (LODs). Koster et al. (2001) reported the first work dealing with the use of MIP coatings on SPME fibers. A silica SPME fiber was silanized, followed by in situ synthesis of the MIP coating on the external surface of the fiber. They prepared the MIP-based SPME fiber using clenbuterol as a template and demonstrated the possibility of selective extraction of brombuterol. This fiber was brittle and the MIP coating stripped during withdrawal of the fiber in the needle. The application of monolithic MIPs with SPME followed by GC was also reported (Djozan \& Baheri, 2007; Djozan \& Ebrahimi, 2008). The MIP was prepared through thermal radical copolymerization of MAA and EDMA in the presence of diacetylmorphine (DAMO) as template, and acetonitrile (ACN) and azobis(isobutyronitrile) (AIBN) were also used as a solvent and an initiator, respectively.

\subsubsection{Molecularly Imprinted Xerogels-based-solid-phase Microextraction}

Despite many advantages, MIPs still need development to overcome limitations such as template leakage, poor accessibility of the binding sites, low binding capacity and non-specific binding (Ansell et al., 1996). The applications of imprinted polymers are also restricted to the use of organic solvents for the dissolution of acrylate or acrylic type polymers which are most commonly used for the preparation of MIPs (Haupt, 2003; Gupta \& Kumar, 2008). To overcome these problems, preparation of MIX by solgel technology has been examined by many researchers (Bagheri \& Piri-Moghadam, 2012; Bagheri et al., 2013d).

Sol-gel imprinting is an emerging field, due to its straightforward synthesis path. Silica-based materials are extremely rigid because of the high degrees of cross-linking in their network. This property is very important and influential in designing and synthesizing the imprinted materials since both the size and shape of the cavities created by the template molecule should be retained after the template removal. High thermal stability of sol-gel derived materials and the possibility of using high temperatures assist the removal process. In addition, sol-gel glasses are structurally porous and can be engineered to have extremely high surface areas. These properties make silica sol-gel matrices an appropriate imprinting host. Figure 2.20 shows a typical process for the imprinting process (Bagheri \& Piri-Moghadam, 2012).

In one study, a simple route for preparation of a novel selective MIX by sol-gel technology was developed. Accordingly, 3-(trimethoxysilyl)propylmethacrylate 

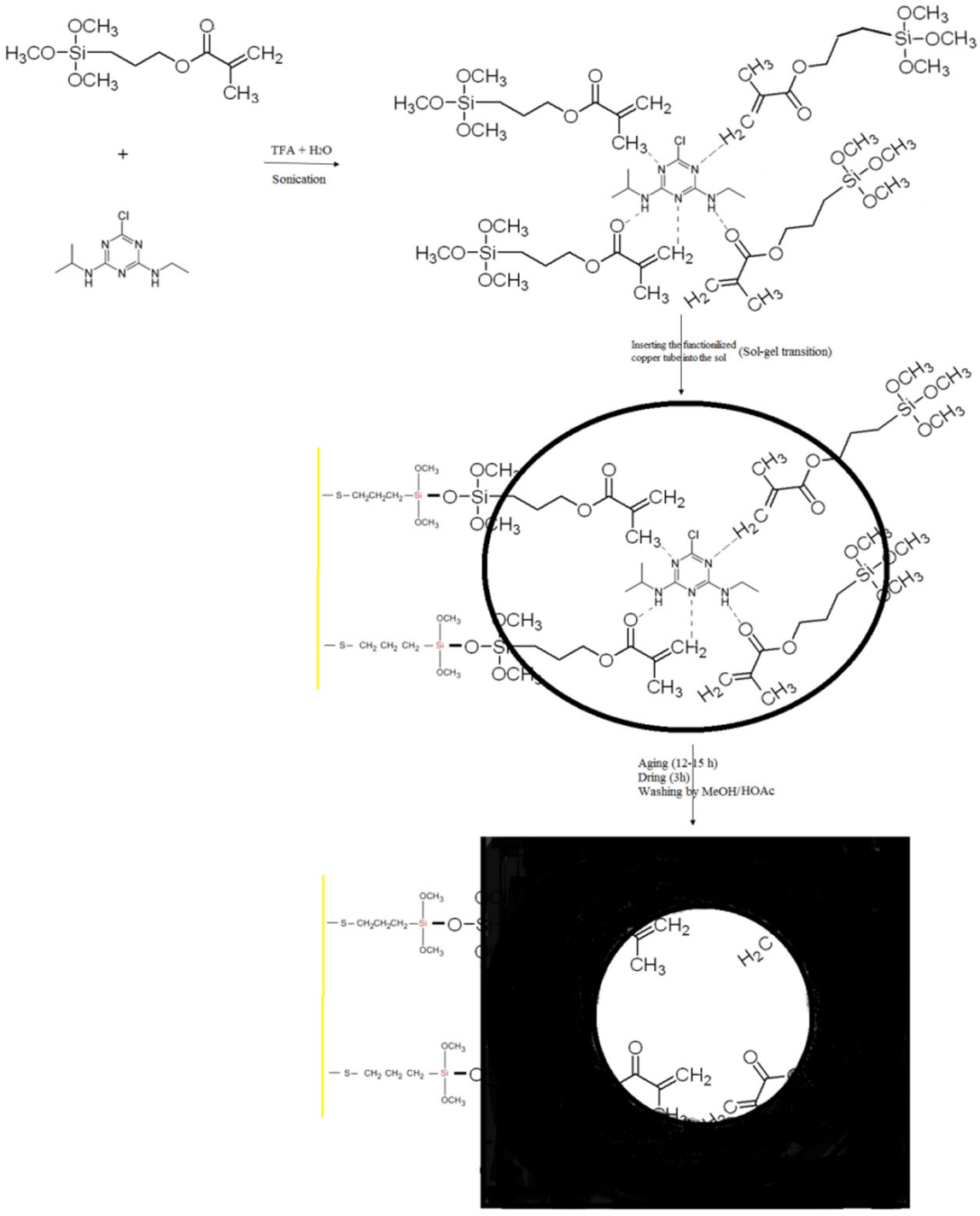

Figure 2.20 A typical process for preparation of MIX of atrazine.

(TMSPMA) was selected as only reagent in the sol-gel process and led to the preparation of a selective sorbent. In TMSPMA, the methacrylate group acts as the functional monomer and the methoxysilane acts as the cross-linker. Atrazine was selected as a model compound for evaluation of the developed method. The prepared MIX showed a high degree of thermal, chemical and mechanical stability along with extensive selectivity towards triazines while a significant reduction in the preparation time was also achieved. 


\subsubsection{Metal-based Coatings}

Because of some shortcomings of commercialized fibers such as breakage of the fused silica fibers, stripping of the coating and damage in organic solvents, many attempts have been made to construct and evaluate fibers with more stability.

\subsubsection{Metal-based Fibers Preparation by Anodization}

It is found by Djozan and co-workers that metal-oxide fiber can be efficient for extracting some organic compounds (Djozan et al., 2001; Djozan \& Abdollahi, 2003). Aluminum wires were anodized by direct current in a solution of sulfuric acid at room temperature. These fibers were used for the extraction of some aliphatic alcohols, benzene, toluene, ethylbenzene and xylenes (BTEX) and petroleum products from gaseous samples. The extraction ability is due to the porous layer of aluminum oxide, which is formed on the metal surfaces (Djozan et al., 2001).

In another work, well-aligned $\mathrm{TiO}_{2}$ nanotubes were grown in situ on a titanium wire substrate by anodization of Ti wire substrates in an electrolyte containing ethylene glycol and $\mathrm{NH}_{4} \mathrm{~F}$. The prepared fiber showed good ability to extract PAHs, anilines, phenols and alkanes from water samples (Liu et al., 2010).

\subsubsection{Metal-based Fibers Developed by Physical Coating}

Different sorbents can be coated onto metal wires and blades using appropriate glues and then used as an SPME fiber. Rastkari et al. reported attachment of single-walled carbon nanotubes (SWCNTs) onto a stainless steel wire by an organic binder and its usage as SPME fiber (Rastkari et al., 2009). Graphene (Ponnusamy \& Jen, 2011) and carbon nanocones (Jiménez-Soto et al., 2010) were also coated onto metal wires. Vuckovic et al. coated silica particles onto the stainless steel wires with a thin layer of Locktite 349 glue. A total of 96 of these fibers were constructed and used for highthroughput extraction of diazepam from blood samples (Vuckovic et al., 2008). In another work, $\mathrm{C}_{18}$ particles were immobilized on the surface of stainless steel blades using biocompatible polyacrylonitrile glue (Mirnaghi et al., 2011). The proposed $\mathrm{C}_{18}$-polyacrylonitrile blade SPME system showed the potential to be employed for high-throughput analysis in a wide variety of research areas. In a recent work, $1.0 \mathrm{~cm}$ pieces of silicon tubing were precisely cut and then coated on the end part of stainless steel wires (Figure 2.21). The prepared fibers were positioned in a homemade PTFEbased constructed 96 hole block to have the possibility of simultaneous immersion of the SPME fibers into the center of individual wells. The constructed high-throughput device was successfully applied for the determination of some selected pesticides in cucumber samples. 


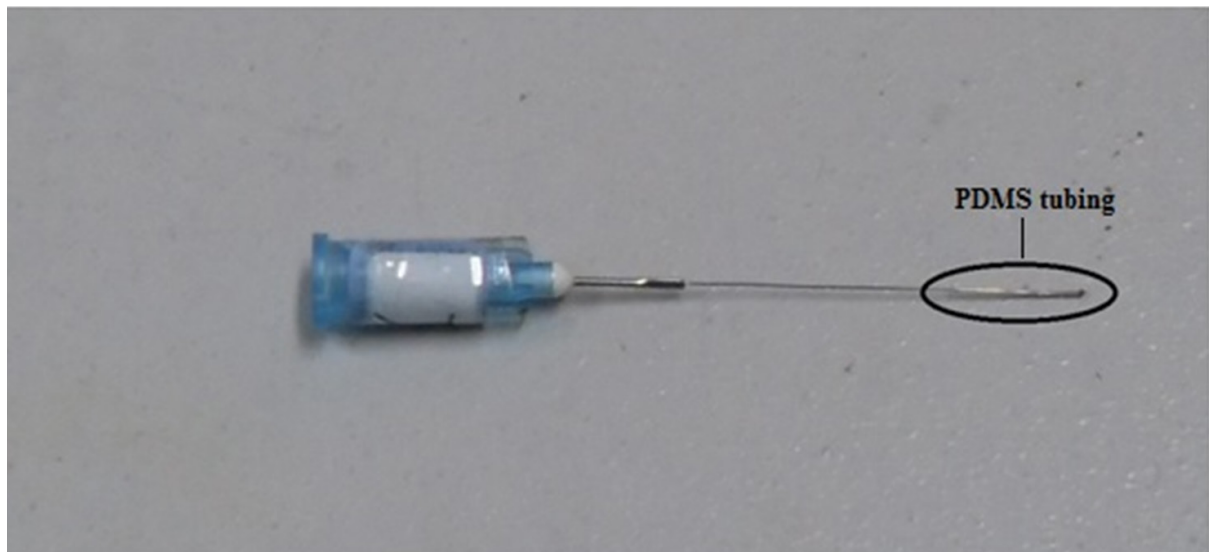

Figure $2.211 .0 \mathrm{~cm}$ of silicon tubing coated on the end of stainless steel wires.

\subsubsection{Metal-based Fibers Developed by Chemical Coating}

Performing the physical coating is easy, but there are some shortcomings like decomposition of glues, fiber swelling and instability in some organic solvents because of the absence of chemical bonding between the sorbent and metal wire. To enhance the coating stability and fiber durability, some efforts were carried out to perform chemical bonding between metal substrate and coating.

Es-haghi et al. (2012) replaced a fused silica substrate by titanium wire which provided high strength and longer fiber life cycle. Titanium isopropoxide was employed as the precursor which provides a sol solution containing $\mathrm{Ti}-\mathrm{OH}$ groups which are similar to the functional groups on the substrate. The similar composition of the sol-gel solution provided more links to the titanium substrate, so the stability and the uniformity of the coating could be greatly improved. The applicability of these fibers was assessed for the HS-SPME of BTEX.

In another work, a gold wire was used as a solid support onto which a film was deposited that consists of a two-dimensional polymer obtained by hydrolysis of a SAM of 3-(trimethoxysilyl)-1-propanthiol (Bagheri et al., 2011b). This first film was covered with a layer of 3-(triethoxysilyl)-propylamine. Next, a stationary phase of oxidized MWCNTs was chemically bound to the surface. The synthetic strategy was verified by Fourier transform infrared spectroscopy and SEM. The fiber was used for HS-SPME of organophosphorus pesticides in water samples.

Pang and Liu reported a novel approach for the fabrication of a SPME fiber by coating a stainless steel fiber with a polymeric ionic liquid (PIL) through covalent bonding (Pang \& Liu, 2012). The stainless steel fiber was sequentially coated with a gold film by a replacement reaction between Fe and Au when immersed in chloroauric acid. A monolayer of 3-(mercaptopropyl) triethoxysilane on the gold layer through the $\mathrm{Au}-\mathrm{S}$ bond was assembled. Eventually, the coating was accomplished with a silica layer by the hydrolysis and polycondensation reaction of the surface-bonded siloxane moieties and the active silicate solution. Then, 1-vinyl-3-(3-triethoxysilylpropyl)- 


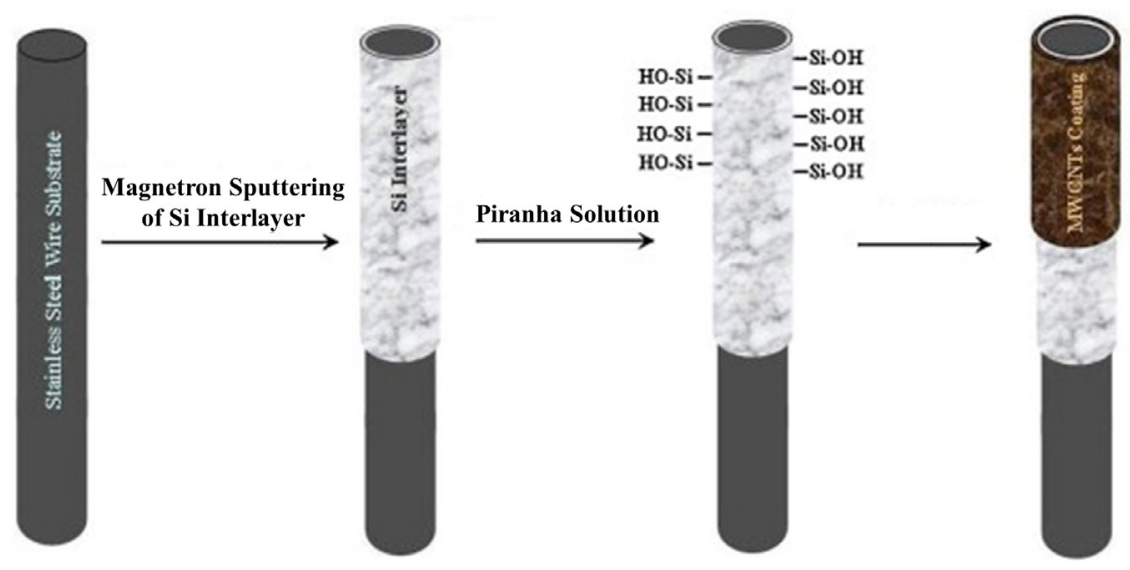

Figure 2.22 Schematic illustration of the preparation of SPME fiber. Reprinted from Liu et al. (2011) with permission from Elsevier. Firstly, a firm Si interlayer was deposited on the stainless steel wire using magnetron sputtering. Then the stainless steel wire with Si interlayer was treated with piranha solution to form $-\mathrm{OH}$ group on the surface. Finally, multilayer-MWCNTs coating was formed on the stainless steel wire by the covalent bond between the active groups on both sides of the substrate and MWCNTs, and the followed van der Waals force-induced spontaneous adsorption of MWCNTs.

4,5-dihydroimidazolium chloride ionic liquid was anchored on the silica layer by covalent bonding, and the PIL film was further formed by free radical copolymerization between 1-vinyl-3-(3-triethoxysilylpropyl)-4,5-dihydroimidazolium and vinylsubstituted imidazolium with AIBN as initiator. The performance of the PIL fiber was evaluated by determination of PAHs in water samples. The developed PIL fiber showed good linearity between 0.5 and $20 \mu \mathrm{g} \mathrm{L}^{-1}$ with regression coefficient in the

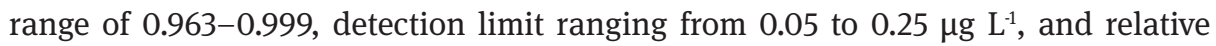
standard deviation of 9.2-29\% $(n=7)$. The developed PIL fiber exhibited comparable analytical performance in compared with a commercial $7 \mu \mathrm{m}$ thickness PDMS fiber in the extraction of PAHs.

In another study, the Si interlayer was prepared using a medium frequency unbalanced magnetron sputtering method in a multifunctional deposition system (Liu et al., 2011). A stainless steel wire was placed in a vacuum chamber and a base pressure of less than $4.0 \times 10^{-3} \mathrm{~Pa}$ was obtained. Prior to the deposition, a $20 \mathrm{~min}$ sputter cleaning in argon plasma was firstly carried out so as to improve the adhesion between the film and substrate. Then, the Si deposition was performed using Ar gas as the sputtering gas, and Si as the target material. The sample holder was kept revolving during the deposition process to ensure the uniformity of the Si layer on the stainless steel wire. The prepared MWCNTs/Si/stainless steel wire fiber (Figure 2.22) not only preserved the excellent SPME behaviors of MWCNTs coatings, but also exhibited a number of 
advantages including high rigidity, long service life and good stability at high temperature and in acid and alkali solutions.

Li et al. (2009) reported the fabrication of a SPME fiber containing platinum coated with SWCNTs that was prepared by electrophoretic deposition and applied to the determination of phenols in aqueous samples. The results revealed that electrophoretic deposition was a simple and reproducible technique for the preparation of SPME fibers coated with SWCNTs without the use of adhesive. The obtained SWCNT coating did not swell in organic solvents or strip off the substrate and also possessed high mechanical strength.

\subsection{Related Techniques}

\subsubsection{Microextraction in Packed Syringe}

Microextraction in packed syringe (MEPS) is a miniaturized version of SPE in which sorbent amounts, sample volumes and desorption solvent volumes are extensively minimized. In this methodology, the tiny sorbent material is inserted either into the barrel of a liquid handling syringe as a plug with polyethylene filters on both sides or between the syringe barrel and the injection needle as a cartridge (Abdel-Rehim, 2010). On-line combination of MEPS with a LC (Abdel-Rehim et al., 2004; Altun et al., 2004) or a GC (Abdel-Rehim et al., 2006; El-Beqqali et al., 2006) can be achieved without any instrumental modification. The environmental or biological sample is drawn through the sorbent by an autosampler (draw and eject in same vial or draw and eject into waste). Similar to SPE, after the entrapment of analytes the solid phase is then washed once by water and/or an acidic solution to remove the proteins and all other possible interfering materials. The analytes are then eluted with an organic solvent such as methanol or the LC mobile phase. A key factor in MEPS is that the volume of solvent used to desorb the analytes from the extracting medium is of a suitable order of magnitude to be injected directly into an LC or GC system. For this purpose, GC systems have to be equipped with a programmed temperature vaporizing (PTV) system. When working with split-splitless injector systems, it is not possible to inject the whole desorption solvent into the injection port. Therefore, it is necessary to use MEPS in an off-line combination with GC systems.

MEPS has found more biologically-oriented applications (Abdel-Rehim, 2004, 2010; Altun et al., 2004) and reports concerning its use in environmental analysis can be found in the literature (El-Beqqali et al., 2006; Prieto et al., 2010; Bagheri, \& Ayazi, 2011). The use of CPs has been implemented as the extracting phase in MEPS for environmental analysis (Bagheri et al., 2013a). For instance, PPy nanowire networks synthesized by a soft template method (Bagheri, \& Ayazi, 2011) were used for MEPS to isolate triazines while $\mathrm{PPy} / \mathrm{PA}$ composite nanofibers, prepared using electrospinning (Bagheri et al., 2012a), were applied as sorbents to extract organophosphorous pes- 
ticides from aqueous media. The PPy nanowire networks were prepared using a soft template technique and its characterization was studied by SEM. The use of micelles in this methodology played an important role in the shape of the growing polymer. The pyrrole monomer was introduced into cetyltrimethylammonium bromide (CTAB) micelles and this led to the formation of nanowires with diameters ranging from 30 to $60 \mathrm{~nm}$. The bulk PPy prepared without CTAB contained spherical particles with diameters of 100-400 nm. The PPy nanowire network was shown to have 7-28 times higher extraction capability in compared with the bulk PPy. Moreover, a polyaniline nanowire network was also prepared by the same method. CTAB was used as structure-directing agent in PANI preparation procedure and this was led to the formation of nanowires with diameters ranging from 35 to $45 \mathrm{~nm}$. The synthesized PANI nanowires network showed higher extraction capability for multiclass pesticides in comparison with the bulk PANI (Bagheri et al., 2012c). In other work, polydiphenylamine (PDPA) nanocomposite reinforced with MWCNTs was applied as a MEPS sorbent for multiresidue determination of pesticides in river waters. This nanocomposite was synthesized by oxidation of diphenylamine in $4 \mathrm{~mol} \mathrm{~L}^{-1}$ sulfuric acid solution containing a fixed amount of CNTs in the presence of CTAB. The effect of CNT doping level and the presence of surfactant on the extraction capability of nanocomposite was investigated and it was revealed that when $4 \%(\mathrm{w} / \mathrm{w})$ of CNT in the presence of CTAB is used, the highest extraction recovery could be achieved (Bagheri et al., 2012b). A headspace adsorptive microextraction technique based on an electrospun polyaniline-nylon-6 (PANI-N6) nanofiber sheet was developed in which the nanofiber diameters were around $200 \mathrm{~nm}$. The novel nanofiber sheet was successfully applied as an extracting medium to isolate some selected chlorobenzenes, as model compounds, from aqueous media (Bagheri, \& Aghakhani, 2012).

For a typical application of MEPS (Bagheri, \& Ayazi, 2011), $1 \mathrm{~mL}$ insulin injection syringes were used. $2 \mathrm{mg}$ of PPy nanowires network was manually inserted inside the syringe between two polyethylene filters (SPE frits, $20 \mu \mathrm{m}$ pore size). For this purpose the size of the SPE frits was changed to match the syringes used. Prior to the first use, the sorbent was manually conditioned with $2 \mathrm{~mL}$ of methanol followed by $4 \mathrm{~mL}$ of water. After that, the spiked sample $(7 \mathrm{~mL})$ was drawn through the syringe forward and backward several times using a variable speed cycling motor which was attached to a circular plate (Figure 2.23). It is important that samples are drawn with sufficient speed to decrease the extraction time and to obtain good percolation between the sample and the solid support. In this study, the speed of the cycling motor was adjusted at $\left.10 \mathrm{rpm}(170 \mu \mathrm{L} \mathrm{s})^{-1}\right)$. After the extraction, the syringe was dried under a flow of nitrogen gas and the analytes were then desorbed with $200 \mathrm{~mL}$ of acetonitrile. The desorption step was performed manually by solvent aspiration into the syringe. Afterward, the desorbed analytes were transferred into a glass vial. Next, the desorbing solvent was evaporated under a nitrogen gas flow until solvent drying was complete. Finally, $10 \mathrm{~mL}$ of acetonitrile was added to the desorption vial and then $2 \mathrm{~mL}$ of desorbed solution was injected into the injection port of the GC system. After each 


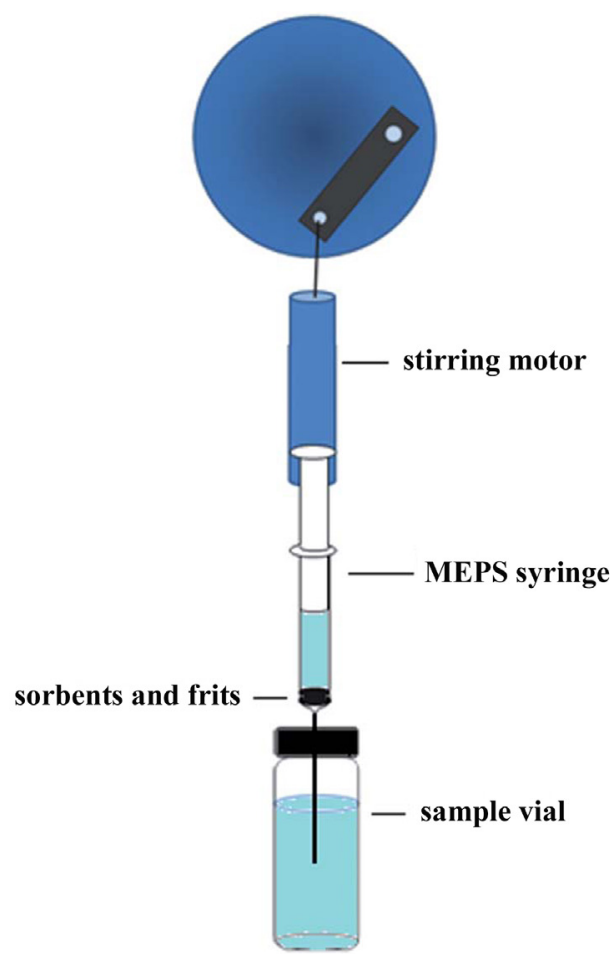

Figure 2.23 Schematic diagram for the extraction set up and MEPS syringe (Bagheri \& Ayazi, 2011).

extraction the MEPS syringe was washed with approximately $200 \mathrm{~mL}$ of acetonitrile, $200 \mathrm{~mL}$ of methanol and $1 \mathrm{~mL}$ of water.

Preparation and characterization of PPy nanowires has also been extensively studied. Template synthesis is a chemical oxidative method which introduces structural directors into the chemical polymerization bath. These structural directors could contain soft templates such as surfactants, organic acids or polyelectrolytes that assist the self-assembly of nanostructures. When the soft-template method is considered, usually micelles or three-dimensional aggregations of micelles are the structure-directing agents. Depending on the specific conditions such as the composition of the surfactant, its concentration, ionic strength, hydrodynamics and temperature, the polymer grows inside or outside the micelles, thus replicating the morphology of the micelles. Most frequently spherical polymer particles are formed. But if the spherical micelles aggregate to form three-dimensionally organized cylindrical structures, then the conditions for the formation of rods can be overcome. In the cases of soft templates it is necessary to remove the corresponding surfactant after termination of the polymerization reaction. The mechanism of formation of the PPy nanowires network could be explained as follows: when pyrrole monomer is added into an aqueous solution containing CTAB and citric acid hydrate, it has been suggested that a three-dimensional network is formed from the assembly or aggregation of pyrrole, 


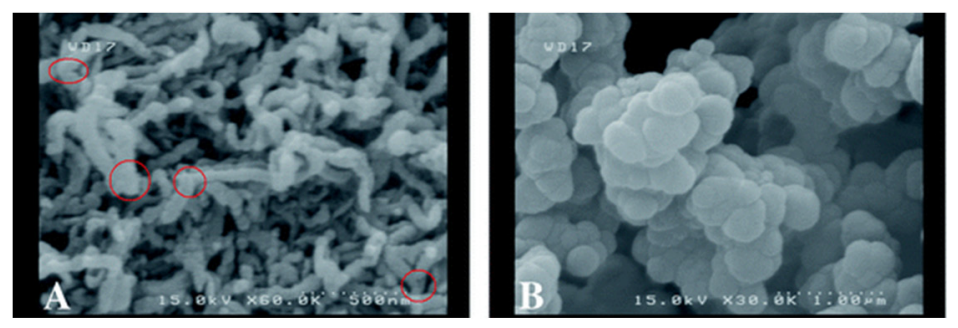

Figure 2.24 SEM image of prepared (A) PPy nanowire network. T junctions are circled on the SEM image (scale bar, $500 \mathrm{~nm}$ ), (B) bulk PPy (scale bar, $1.00 \mu \mathrm{m}$ ) (Bagheri \& Ayazi, 2011).

$\mathrm{CTAB}$, and citric acid before the oxidation polymerization of pyrrole occurs. Based on these results, it can be concluded that when CTAB, citric acid, and pyrrole are added to water, micelles would be formed because of the long alkyl chain of CTAB. Two micelles of CTAB might be connected by citric acid molecules to form one or more $\mathrm{T}$ junctions. When APS is added into the reaction solution, the three-dimensional networks of the soft templates can be elongated and form the three dimensional PPy nanowires network. Also, CI acts as a dopant for PPy in this procedure and it is not a prerequisite for wire formation, but the ability of the micelles to link together to form a micelle network is improved when the PPy is formed after addition of the oxidant APS. The surface characteristics of the prepared polypyrrole nanowires were investigated by SEM. According to Figure 2.24A, the formation of nanowires is confirmed and all of them are packed in rough porous structures. Also, it is shown that the nanowire diameters are in the range of $30-60 \mathrm{~nm}$ and there are some observable $\mathrm{T}$ junctions as discussed above.

The SEM image of bulk PPy (CTAB was not used in preparation of this polymer) is illustrated in Figure 2.24B. Clearly, the PPy prepared without CTAB are all spherical particles with diameters of $100-400 \mathrm{~nm}$, indicating that these particles are linked and packed together.

Analysis of the extraction capability of the prepared PPy nanowires network was also carried out. Features such as high surface area and $\pi$-functional groups of the $\mathrm{CP}$, together with polar functional groups of CI, are important characteristics of the fabricated PPy nanowire network which make it a suitable candidate for extraction purposes. To investigate the extraction capability of the prepared sorbent, the MEPS syringes were prepared using the PPy nanowires and the bulk PPy. The results revealed that the PPy nanowires network exhibits 7 to 28 times higher extraction capability in comparison with that of the bulk PPy. This enhancement in extraction capability is probably due to the increase in surface area and hydrophilicity of the PPy nanowires network. To investigate the extraction capability of the prepared PPy nanowires, the MEPS syringes were prepared using two different kinds of PPy.

These examples demonstrate that MEPS is suitable for analysis of pollutants and pesticides in water samples as well as drugs in biofluids. The matrix effect is rather 
insignificant as far as the water sample analysis is concerned while this strategy has already proven to be quite suitable for biofluids. Furthermore, employing such a miniaturized extraction scale meets the demands of reduced solvent consumption and waste production.

\subsubsection{Stir Bar Sorptive Extraction}

Stir bar sorptive extraction (SBSE) was developed in 1999 by Sandra and co-workers (Baltussen et al., 1999) and commercialized under the name "Twister". SBSE is able to extract and preconcentrate the desired compounds from liquid matrices without the use of solvents (David et al., 2003a, 2003b). SBSE has been applied successfully to trace analysis of environmental samples and has satisfactory analytical reproducibility for the determination of volatile and semi-volatile components of biological mixtures. In SBSE, organic analytes are enriched from aqueous samples by sorption onto a thick film of PDMS coated onto a glass-enveloped magnet (Mitra, 2003; Sánchez-Rojas et al., 2009). The sample extraction takes place during stirring for a fixed time. The spin bar is then removed and placed in a glass tube, which is transferred into a thermal desorption system where the analytes are thermally recovered and analyzed on-line either by GC or LC. Similar to other miniaturized sorptive extraction systems, SBSE is an equilibrium-based technique and the analyte extraction from the aqueous phase into the extracting phase is controlled by the partitioning coefficient of the analyte between PDMS and the aqueous phase. Detailed studies have correlated this partition coefficient with the octanol-water distribution coefficient $\left(K_{\text {OW }}\right)$.

Using PDMS as the sorbent, the primary mechanism of interaction with organic analytes is via absorption or partitioning into the PDMS coating such that the distribution constant (equation 2.6) between PDMS and water $\left(K_{P D M S / W}\right)$ is proposed to be proportional to the octanol-water partition coefficient $\left(K_{O W}\right)$ (Baltussen et al., 1999):

$K_{D}=\frac{[X]_{B}}{[X]_{A}}=K_{P D M S W} \approx K_{O W}$

According to the theoretical development for this technique (Baltussen et al., 1999):

$K_{P D M S W} \approx K_{O W}=\frac{[X]_{P D M S}}{[X]_{W}}=\frac{m_{P D M S}}{m_{W}} x \frac{V_{W}}{V_{P D M S}}$

where $[X]_{P D M S}$ and $[X]_{W}$ and $m_{P D M S}$ and $m_{W}$ are the analyte concentration and the analyte mass in the PDMS and water phase, respectively, while $V_{P D M S}$ and $V_{W}$ represent the volume of the PDMS sorbent and water phase, respectively. Therefore, the parameters determining the mass of an analyte recovered by SBSE using the PDMS sorbent are the partition coefficient of the analyte $\left(K_{O W}\right)$ and the phase ratio $\left(V_{W} / V_{P D M S}\right)$ of the volume of the water phase to the volume of the PDMS coating on the stir bar. 
In general, SBSE is considered to be superior to SPME in terms of sensitivity and accuracy for determining trace level quantities in difficult matrices. The amount of analyte extracted is proportional to the volume of the extraction phase, which in SPME is very small. Hence the detection limits of a method can be improved by increasing the volume of the extraction phase. This can be achieved by increasing the film thickness, either on a fiber support or by using a multifiber method. However, much longer equilibration time is required because the extraction rate is controlled by the diffusion from the sample matrix through the boundary layer into the extraction phase. SBSE is advantageous in dealing with very dilute media and trace concentration samples; compared to SPME, SBSE generally yields better detection limits. On the other hand, SPME can be fully automated which is not yet completely true for SBSE. It has been demonstrated that a wide range of volatile and semi-volatile substances such as phenylethyl alcohol, 1-ethenyl- 4-methoxybenzene, 2-phenylethyl acetate, 1-(ethylthio)-2-methylbenzene, 3,7-dimethyl-2,6-octadienoic acid, methyl ester, $\alpha$-cubelene, copaene, terpenes, PCBs and pesticides can be retained on a polymercoated magnetic bar (Baltussen et al., 1999, 2002; David et al., 2003a, 2003b; Soini et al., 2005). The amount of PDMS coating on a stir bar can be precisely controlled, increasing the reproducibility and reliability of extraction. A typical polymer phase volume of $24-100 \mu \mathrm{L}$ in SBSE well exceeds that of SPME (typically, $0.5 \mu \mathrm{L}$ ). By rapid magnetic stirring of the aqueous medium, equilibrium on the PDMS layer is ensured during sorption so that the analyte concentration is constant and reproducible. PDMS sorption-based analyses have been described in environmental samples, essential oils, foodstuffs and beverages. Similar techniques for human biological samples have been reported. A number of new applications of SBSE and other extraction procedures have been published. These improvements involve SBSE with in situ derivatization, SBSE with in situ de-conjugation, TD in the multi-shot mode and TD with in tube derivatization method. These methods were applied successfully to the trace analysis of environmental and biological samples. (Kawaguchi et al., 2006; Quintana \& Rodríguez, 2006; Buchberger \& Zaborsky, 2007; David \& Sandra, 2007; Picó et al., 2007).

\subsubsection{Needle Trap Extraction}

In the last few years, the inside needle capillary adsorption trap (INCAT) technique emerged as a solventless sample preparation approach and alternative extraction method derived from SPME. Two approaches could be associated with this technique. In the first one, the sorbent is placed as adsorbent layer, acting as non-polar or polar phase, on the interior surface of a needle (Musshoff et al., 2003). In the first approach, Murphy described a technique based on the use of an internally coated hollow needle (Murphy, 1996). Shojania et al. have used an adsorbing carbon coating on the interior surface of a hollow stainless steel needle as an INCAT device (Shojania et al., 1999). Later, a similar approach called "solid-phase dynamic extraction (SPDE)" was suc- 
cessfully applied to the analysis of pesticides in water (Lipinski, 2001) and amphetamines and synthetic design drugs in hair (Musshoff et al., 2002).

The other category is in-needle packing with commercially available sorbents or chemically synthesized compounds. The needle-trap devices are inexpensive, robust and reusable, and are suitable for the sampling and analysis of volatile organic compounds from many different sample matrices. Compared to the fragile silica-based SPME fiber, the needle device is rather impossible to break mechanically. Moreover, the extraction medium possesses a large extraction phase volume and a high preconcentration ability. Similar to SPME, needle-like devices are particularly convenient for automation and development of on-line procedures (Bagheri et al., 2009, 2011a).

\subsection{Conclusions}

SPME, since its introduction in the early 90's, has shown to have inherent potential to be adapted as a powerful technique in sample preparation methodologies. This versatile technique, together with other relevant methods, has been extended in many aspects of technical, physical and chemical points of view. Selective chemical deposition approaches have led to the emerging methodologies in which most analytical demands are almost met. The implementation of nanomaterials, sol-gel technology and metal-based coatings in SPME and other related techniques are developments which led to more stable, precise and accurate analysis while ease of operation and lower costs are also encountered. It is anticipated that SPME and other relevant techniques will play a major role in the future development of sample preparation.

\section{Abbreviations}

\begin{tabular}{|c|c|}
\hline$\mu$-SPE & micro-solid phase extraction \\
\hline 2-ATP & 2-aminothiophenol \\
\hline 3-ATP & 3-aminothiophenol \\
\hline $\mathrm{ACN}$ & acetonitrile \\
\hline AIBN & azobis(isobutyronitrile) \\
\hline APS & ammonium persulfate \\
\hline BFEE & boron trifluoride diethyl etherate \\
\hline BMIPF $_{6}$ & 1-butyl-3-methylimidazolium hexafluorophosphate \\
\hline BPANI & polyaniline-1-butyl-3-methylimidazolium hexafluorophosphate composite \\
\hline BTEX & benzene, toluene, ethylbenzene and xylenes; butyronitrile \\
\hline CME & capillary microextraction \\
\hline CNTs & carbon nanotubes \\
\hline CPs & conductive polymers \\
\hline CTAB & cetyltrimethylammonium bromide \\
\hline
\end{tabular}




$\begin{array}{ll}\text { CV } & \text { cyclic voltammetry } \\ \text { DAMO } & \text { diacetylmorphine } \\ \text { DS } & \text { dodecylsulfate } \\ \text { EDMA } & \text { ethylene glycol dimethacrylate } \\ \text { ESI } & \text { electrospray ionization } \\ \text { G } & \text { grapheme } \\ \text { GC } & \text { gas chromatography } \\ \text { GO } & \text { graphene oxide } \\ \text { HPLC } & \text { high performance liquid chromatography } \\ \text { HS-SDME } & \text { headspace solid-phase microextraction } \\ \text { INCAT } & \text { inside needle capillary adsorption trap } \\ \text { LC } & \text { liquid chromatography } \\ \text { LOD } & \text { limit of detection } \\ \text { MAA } & \text { methacrylic acid } \\ \text { m-ABA } & \text { m-aminobenzoic acid } \\ \text { MEPS } & \text { microextraction in a packed syringe } \\ \text { MIP } & \text { molecular imprinted polymer } \\ \text { MIX } & \text { molecular imprinted xerogel } \\ \text { MS } & \text { mass spectrometry } \\ \text { MWCNTs } & \text { multi-walled carbon nanotubes } \\ \text { NiPcTS } & \text { tetrasulfonated nickel phthalocyanine } \\ \text { OCPs } & \text { organochlorine pesticides } \\ \text { ORMOSIL } & \text { organically modified silica } \\ \text { Pac } & \text { polyacetylene } \\ \text { PANI } & \text { polyaniline } \\ \text { PATP } & \text { polyaminothiophenol } \\ \text { PBDEs } & \text { polybrominated diphenyl ethers } \\ \text { PCBs } & \text { polychlorinated biphenyls } \\ \text { PFOS-PANI perfluorooctanesulfonic acid-doped PANI } \\ \text { PDPA } & \text { polydiphenylamine } \\ \text { PIL } & \text { polymeric ionic liquid } \\ \text { PMME } & \text { polymer monolith microextraction } \\ \text { PP } & \text { polyphosphate } \\ \text { PPy } & \text { polypyrrole } \\ \text { PPy-S } & \text { sulfate-doped PPy } \\ \text { PSS } & \text { poly(styrenesulfonate) } \\ \text { PTFE } & \text { Polytetrafluoroethylene } \\ \text { PTh } & \text { polythiophene } \\ \text { PELDI } & \text { programmed temperature vaporizing } \\ \end{array}$




$\begin{array}{ll}\text { SEM } & \text { scanning electron microscopy } \\ \text { SPDE } & \text { solid-phase dynamic extraction } \\ \text { SPE } & \text { solid phase extraction } \\ \text { SPME } & \text { solid-phase microextraction } \\ \text { SWCNTs } & \text { single-walled carbon nanotubes } \\ \text { TD } & \text { thermal desorption } \\ \text { TEOS } & \text { tetraethoxysilane } \\ \text { TFME } & \text { thin film microextraction } \\ \text { TMOS } & \text { tetramethoxysilane } \\ \text { TMSPMA } & \text { 3-(trimethoxysilyl)propylmethacrylate } \\ \text { TOS } & \text { toluene-p-sulfonate } \\ \text { VOCs } & \text { volatile organic compounds. }\end{array}$

\section{References}

Abdel-Rehim, M. (2004). New trend in ample preparation: on line microextraction in packed syringe for liquid and gas chromatography applications: I. Determination of local anaesthesics in human plasma samples using gas chromatography-mass spectrometry. Journal of Chromatography B: Analytical Technologies in the Biomedical and Life Sciences, 801, 317-321.

Abdel-Rehim, M. (2010). Recent advances in microextraction by packed sorbent for bioanalysis. Journal of Chromatography A, 1217, 2569-2580.

Abdel-Rehim, M., Altun, Z., \& Blomberg, L. (2004). Microextraction in packed syringe (MEPS) for liquid an gas chromatographic applications. Part II - Determination of ropivacaine and its metabolites in human plasma samples using MEPS with liquid chromatography/tandem mass spectrometry. Journal of Mass Spectrometry, 39, 1488-1493.

Abdel-Rehim, M., Dahlgren, M., Claude, S., Tabacchi, R., \& Blomberg, L. (2006). Microextraction in packed syringe (MEPS) utilizing methylcyanopropyl-silarylene as coating polymer for extraction of drugs in biological samples. Journal of Liquid Chromatography \& Related Technology, 29, 2537-2544.

Ahlrichs, J. L., Serna, C., \& Serratosa, J. M. (1975). Structural hydroxyls in sepiolites. Clays and Clay Minerals, 23, 119-124.

Akelah, A., Salahuddin, N., Hiltner, A., Baer, E., \& Moet, A. (1994). Morphological hierarchy of butadieneacrylonitrile/montmorillonite nanocomposite. Nanostructured Materials, 4, 965-978.

Altun, Z., Abdel-Rehim M., \& Blomberg, L.G. (2004). New trends in sample preparation: On-line microextraction in packed syringe (MEPS) for LC and GC applications: Part III: Determination and validation of local anaesthesics in human plasma samples using a cation-exchange sorbent, and MEPS-LC-MS-MS. Journal of Chromatography B: Analytical Technologies in the Biomedical and Life Sciences, 813, 129-135.

Ansell, R. J., Kriz, D., \& Mosbach, K. (1996). Molecularly imprinted polymers for bioanalysis: Chromatography, binding assays and biomimetic sensors. Current Opinion in Biotechnology, 7, 89-94.

Asadollahzadeh, H., Noroozian, E., \& Maghsoudi, S. (2010). Solid-phase microextraction of phthalate esters from aqueous media by electrochemically deposited carbon nanotube/ polypyrrole composite on a stainless steel fiber. Analytica Chimica Acta, 669, 32-38.

Bagheri, H., \& Aghakhani, A. (2011). Novel nanofiber coatings prepared by electrospinning technique for headspace solid-phase microextraction of chlorobenzenes from environmental samples. Analytical Methods, 3, 1284-1289. 
Bagheri, H., \& Aghakhani, A. (2012). Polyaniline-nylon-6 electrospun nanofibers for headspace adsorptive microextraction. Analytica Chimica Acta, 713, 63-69.

Bagheri, H., \& Ayazi, Z. (2011). Polypyrrole nanowires network for convenient and highly efficient microextraction in packed syringe. Analytical Methods, 3, 2630-2636.

Bagheri, H., \& Piri-Moghadam, H. (2012). Sol-gel-based molecularly imprinted xerogel for capillary microextraction. Analytical and Bioanalytical Chemistry, 404, 1597-1602.

Bagheri, H., \& Roostaie, A. (2012). Aniline-silica nanocomposite as a novel solid phase microextraction fiber coating. Journal of Chromatography A, 1238, 22-29.

Bagheri, H., Ayazi, Z., \& Aghakhani, A. (2011a). A novel needle trap sorbent based on carbon nanotube-sol-gel for microextraction of polycyclic aromatic hydrocarbons from aquatic media. Analytica Chimica Acta, 683, 212-220.

Bagheri, H., Ayazi, Z., \& Naderi, M. (2013a). Conductive polymer-based microextraction methods: A review. Analytica Chimica Acta, 767, 1-13.

Bagheri, H., Ayazi, Z., \& Sistani, H. (2011b). Chemically bonded carbon nanotubes on modified gold substrate as novel unbreakable solid phase microextraction fiber. Microchimica Acta, 174, 295-301.

Bagheri, H., Ayazi, Z., Aghakhani, A., \& Alipour, N. (2012a). Polypyrrole/polyamide electrospunbased sorbent for microextraction in packed syringe of organophosphorous pesticides from aquatic samples. Journal of Separation Science, 35, 114-120.

Bagheri, H., Ayazi, Z., Es'haghi, A., \& Aghakhani, A. (2012b). Reinforced polydiphenylamine nanocomposite for microextraction in packed syringe of various pesticides. Journal of Chromatography $A, 1222,13-21$.

Bagheri, H., Babanezhad, E., \& Es-haghi, A. (2007). An aniline-based fiber coating for solid phase microextraction of polycyclic aromatic hydrocarbons from water followed by gas chromatography-mass spectrometry. Journal of Chromatography A, 1152, 168-174.

Bagheri, H., Babanezhad, E., \& Khalilian, F. (2009). An interior needle electropolymerized pyrrolebased coating for headspace solid-phase dynamic extraction. Analytica Chimica Acta, 634, 209-214.

Bagheri, H., Bayat, P., \& Piri-Moghadam, H. (2013b). Grafting the sol-gel based sorbents by diazonium salts: A novel approach toward unbreakable capillary microextraction. Journal of Chromatography A, 1318, 58-64.

Bagheri, H., Es-haghi, A., \& Rouini, M. R. (2005a). Sol-gel-based solid-phase microextraction and gas chromatography-mass spectrometry determination of dextromethorphan and dextrorphan in human plasma. Journal of Chromatography B: Analytical Technologies in the Biomedical and Life Sciences, 818, 147-157.

Bagheri, H., Es'haghi, A., Es-haghi, A., \& Mesbahi, N. (2012c). A high-throughput approach for the determination of pesticide residues in cucumber samples using solid-phase microextraction on 96-well plate. Analytica Chimica Acta, 740, 36-42.

Bagheri, H., Es'haghi, A., Es-haghi, A., \& Mohammadkhani, E. (2013c). High-throughput micro-solid phase extraction on 96-well plate using dodecyl methacrylate-ethylen glycol dimethacrylate monolithic copolymer. Analytica Chimica Acta, 792, 59-65.

Bagheri, H., Mir, A., \& Babanezhad, E. (2005b). An electropolymerized aniline-based fiber coating for solid phase microextraction of phenols from water. Analytica Chimica Acta, 532, 89-95.

Bagheri, H., Piri-Moghadam, H., \& Ahdi, T. (2012d). Role of precursors and coating polymers in sol-gel chemistry toward enhanced selectivity and efficiency in solid phase microextraction. Analytica Chimica Acta, 742, 45-53.

Bagheri, H., Piri-Moghadam, H., \& Es'haghi, A. (2011c). An unbreakable on-line approach towards sol-gel capillary microextraction. Journal of Chromatography A, 1218, 3952-3957. 
Bagheri, H., Piri-Moghadam, H., \& Naderi, M. (2012e). Towards greater mechanical, thermal and chemical stability in solid-phase microextraction. TrAC Trends in Analytical Chemistry, 34, 126-138.

Bagheri, H., Piri-Moghadam, H., Bayat, P., \& Balalaie, S. (2013d). Application of sol-gel based molecularly imprinted xerogel for on-line capillary microextraction of fentanyl from urine and plasma samples. Analytical Methods, 5, 7096-7101.

Bagheri, H., Sistani, H., \& Ayazi, Z. (2011d). Novel unbreakable solid-phase microextraction fiber by electrodeposition of silica sol-gel on gold. Journal of Separation Science, 34, 3246-3252.

Bain, C. D., Troughton, E. B., Tao, Y. T., Evall, J., Whitesides, G. M., \& Nuzzo, R. G. (1989). Formation of monolayer films by the spontaneous assembly of organic thiols from solution onto gold. Journal of the American Chemical Society, 111, 321-335.

Baltussen, E., Cramers, C.A., \& Sandra, P. (2002). Sorptive sample preparation - A review. Analytical \& Bioanalytical Chemistry, 373, 3-22.

Baltussen, E., Sandra, P., David, F., \& Cramers, C. (1999). Stir bar sorptive extraction (SBSE), a novel extraction technique for aqueous samples: Theory and principles. Journal of Microcolumn Separation, 1999, 11, 737-747.

Bhardwaj, N., \& Kundu, S. C. (2010). Electrospinning: A fascinating fiber fabrication technique. Biotechnology Advances, 28, 325-347.

Blinova, N. V., Stejskal, J., Trchová, M., \& Prokeš, J. (2006). Polyaniline prepared in solutions of phosphoric acid: Powders, thin films, and colloidal dispersions. Polymer, 47, 42-48.

Boukerma, K., Chehimi, M. M., Pinson, J., \& Blomfield, C. (2003). X-ray photoelectron spectroscopy evidence for the covalent bond between an iron surface and aryl groups attached by the electrochemical reduction of diazonium salts. Langmuir, 19, 6333-6335.

Breton, T., \& Bélanger, D. (2008). Modification of carbon electrode with aryl groups having an aliphatic amine by electrochemical reduction of in situ generated diazonium cations. Langmuir, 24, 8711-8718.

Buchberger, W., \& Zaborsky, P. (2007). Sorptive extraction techniques for trace analysis of organic pollutants in the aquatic environment. Acta Chimica Slovenica, 54, 1-13.

Cao, D. d., Lü, J. x., Liu, J. f., \& Jiang, G. b. (2008). In situ fabrication of nanostructured titania coating on the surface of titanium wire: A new approach for preparation of solid-phase microextraction fiber. Analytica Chimica Acta, 611, 56-61.

Chen, L., Chen, W., Ma, C., Du, D., \& Chen, X. (2011). Electropolymerized multiwalled carbon nanotubes/polypyrrole fiber for solid-phase microextraction and its applications in the determination of pyrethroids. Talanta, 84, 104-108.

Chen, M. L., Wei, S. S., Yuan, B. F., \& Feng, Y. Q. (2012). Preparation of methacrylate-based monolith for capillary hydrophilic interaction chromatography and its application in determination of nucleosides in urine. Journal of Chromatography A, 1228, 183-192.

Chen, S. A., \& Tsai, C. C. (1993). Structure/properties of conjugated conductive polymers. 2. 3-ethersubstituted polythiophenes and poly(4-methylthiophenes). Macromolecules, 26, 2234-2239.

Chigome, S., Darko, G., Buttner, U., \& Torto, N. (2010). Semi-micro solid phase extraction with electrospun polystyrene fiber disks. Analytical Methods, 2, 623-626.

Chong, S. L., Wang, D., Hayes, J. D., Wilhite, B. W., \& Malik, A. (1997). Sol-gel coating technology for the preparation of solid-phase microextraction fibers of enhanced thermal stability. Analytical Chemistry, 69, 3889-3898.

Corriu, R., \& Trong Anh, H. (2009). Molecular Chemistry of Sol-Gel Derived Nanomaterials. UK: John Wiley \& Sons.

David, F., \& Sandra, P. (2007). Stir bar sorptive extraction for trace analysis. Journal of Chromatography A, 1152, 54-69.

David. F., Tienpont, B., \& Sandra, P. (2003a). Stir-bar sorptive extraction of trace organic compounds from aqueous matrices. LCGC North America, 21, 108-118. 
David. F., Tienpont, B., \& Sandra, P. (2003b). Stir-bar sorptive extraction of trace organic compounds from aqueous matrices. LCGC Europe, 16, 410-417.

Deng, D. L., Zhang, J. Y., Chen, C., Hou, X. L., Su, Y. Y., \& Wu, L. (2012). Monolithic molecular imprinted polymer fiber for recognition and solid phase microextraction of ephedrine and pseudoephedrine in biological samples prior to capillary electrophoresis analysis. Journal of Chromatography A, 1219, 195-200.

Djozan, D., \& Abdollahi, L. (2003). Anodized zinc wire as a solid-phase microextraction fiber. Chromatographia, 57, 799-804.

Djozan, D., \& Bahar, S. (2004). Solid-phase microextraction of aliphatic alcohols based on polyaniline coated fibers. Chromatographia, 59, 95-99.

Djozan, D., \& Baheri, T. (2007). Preparation and evaluation of solid-phase microextraction fibers based on monolithic molecularly imprinted polymers for selective extraction of diacetylmorphine and analogous compounds. Journal of Chromatography A, 1166, 16-23.

Djozan, D., \& Ebrahimi, B. (2008). Preparation of new solid phase micro extraction fiber on the basis of atrazine-molecular imprinted polymer: Application for GC and GC/MS screening of triazine herbicides in water, rice and onion. Analytica Chimica Acta, 616, 152-159.

Djozan, D., Assadi, Y., \& Haddadi, S. H. (2001). Anodized aluminum wire as a solid-phase microextraction fiber. Analytical Chemistry, 73, 4054-4058.

Djozan, D., Pournaghi-Azar, M. H., \& Bahar, S. (2004). Modified polypyrrole with tetrasulfonated nickel phthalocyanine as a fiber for solid-phase microextraction. Application to the extraction of BTEX compounds from water samples. Chromatographia, 59, 595-599.

Du, W., Zhao, F., \& Zeng, B. (2009). Novel multiwalled carbon nanotubes-polyaniline composite film coated platinum wire for headspace solid-phase microextraction and gas chromatographic determination of phenolic compounds. Journal of Chromatography A, 1216, 3751-3757.

Ebelmann, M. (1846). Sur les combinaisons des acides borique et silicique avec les ethers. Annales de Chimie et de Physique, 16, 129-166.

Ebelmann, M. (1847). Sur l'hyalite artificielle et l'hydrophane. Les Comptes rendus de l'Académie des sciences, 25, 854-856.

Eisert, R., \& Levsen, K. (1995). Determination of pesticides in aqueous samples by solid-phase microextraction in-line coupled to gas chromatography-mass spectrometry. Journal of the American Society for Mass Spectrometry, 6, 1119-1130.

El-Beqqali, A., Kussak, A., \& Abdel-Rehim, M. (2006). Fast and sensitive environmental analysis utilizing microextraction in packed syringe online with gas chromatography-mass spectrometry. Determination of polycyclic aromatic hydrocarbons in water. Journal of Chromatography A, 1114, 234-238.

Es-haghi, A., Hosseini, S. M., \& Khoshhesab, Z. M. (2012). Development and application of a new solid-phase microextraction fiber by sol-gel technology on titanium wire. Analytica Chimica Acta, 742, 74-79.

Fan, Y., Feng, Y. Q., Da, S. L., \& Shi, Z. G. (2004). Poly (methacrylic acid-ethylene glycol dimethacrylate) monolithic capillary for in-tube solid phase microextraction coupled to high performance liquid chromatography and its application to determination of basic drugs in human serum. Analytica Chimica Acta, 523, 251-258.

Fontanals, N., Marcé, R. M., \& Borrull, F. (2007). New materials in sorptive extraction techniques for polar compounds. Journal of Chromatography A, 1152, 14-31.

Freund, M. S., \& Deore, B. A. (2007). Self Doped Conducting Polymers. UK: John Wiley \& Sons.

Friedrich, K., Fakirov, S., \& Zhang, Z. (2005). Polymer Composite: From Nano-to Macro-Scale. New York: Springer.

Gao, H., Jiang, T., Han, B., Wang, Y., Du, J., Liu, Z., et al. (2004). Aqueous/ionic liquid interfacial polymerization for preparing polyaniline nanoparticles. Polymer, 45, 3017-3019. 
Gao, Z., Li, W., Liu, B., Liang, F., He, H., Yang, S., et al. (2011). Nano-structured polyaniline-ionic liquid composite film coated steel wire for headspace solid-phase microextraction of organochlorine pesticides in water. Journal of Chromatography A, 1218, 6285-6291.

Ghassempour, A., Najafi, N. M., Mehdinia, A., Davarani, S. S. H., Fallahi, M., \& Nakhshab, M. (2005). Analysis of anatoxin-a using polyaniline as a sorbent in solid-phase microextraction coupled to gas chromatography-mass spectrometry. Journal of Chromatography A, 1078, 120-127.

Ghosh, S. K. (2006). Functional Coatings. Weinheim: Wiley-VCH.

Gómez-Hens, A., Fernández-Romero, J. M., \& Aguilar-Caballos, M. P. (2008). Nanostructures as analytical tools in bioassays. TrAC Trends in Analytical Chemistry, 27, 394-406.

Gratzl, M., Hsu, D. F., Riley, A. M., \& Janata, J. (1990). Electrochemically deposited polythiophene.

1. Ohmic drop compensation and the polythiophene paradox. Journal of Physical Chemistry, 94, 5973-5981.

Gupta, R., \& Kumar, A. (2008). Molecular imprinting in sol-gel matrix. Biotechnology Advances, 26, 533-547.

Haupt, K. (2003). Imprinted polymers - Tailor-made mimics of antibodies and receptors. Chemical Communications, 9, 171-177.

Havenga, W. J., \& Rohwer, E. R. (1999). Chemical characterization and screening of hydrocarbon pollution in industrial soils by headspace solid-phase microextraction. Journal of Chromatography A, 848, 279-295.

Huang, Z. M., Zhang, Y. Z., Kotaki, M., \& Ramakrishna, S. (2003). A review on polymer nanofibers by electrospinning and their applications in nanocomposites. Composites Science and Technology, 63, 2223-2253.

Jiménez-Soto, J. M., Cárdenas, S., \& Valcárcel, M. (2010). Carbon nanocones/disks as new coating for solid-phase microextraction. Journal of Chromatography A, 1217, 3341-3347.

Kabir, A., Furton, K. G., \& Malik, A. (2013). Innovations in sol-gel microextraction phases for solvent-free sample preparation in analytical chemistry. TrAC Trends in Analytical Chemistry, 45, 197-218.

Kang, X., Pan, C., Xu, Q., Yao, Y., Wang, Y., Qi, D., et al. (2007). The investigation of electrospun polymer nanofibers as a solid-phase extraction sorbent for the determination of trazodone in human plasma. Analytica Chimica Acta, 587, 75-81.

Karpovich, D. S., Schessler, H. M., \& Blanchard, G. J. (1998). Self-Assembled Monolayers of Thiols (Vol. 24). New York: Academic Press.

Kataoka, H. (2011). Current developments and future trends in solid-phase microextraction techniques for pharmaceutical and biomedical analyses. Analytical Sciences, 27, 893-905.

Kataoka, H., Ishizaki, A., Nonaka, Y., \& Saito, K. (2009). Developments and applications of capillary microextraction techniques: A review. Analytica Chimica Acta, 655, 8-29.

Kawaguchi, M., Ito, R., Saito, K., \& Nakazawa, H. (2006). Novel stir bar sorptive extraction methods for environmental and biomedical analysis. Journal of Pharmacutical \& Biomedical Analysis, 40, 500-508.

Kawasumi, M., Hasegawa, N., Usuki, A., \& Okada, A. (1999). Liquid crystal/clay mineral composites. Applied Clay Science, 15, 93-108.

Koster, E. H. M., Crescenzi, C., Den Hoedt, W., Ensing, K., \& De Jong, G. J. (2001). Fibers coated with molecularly imprinted polymers for solid-phase microextraction. Analytical Chemistry, 73, 3140-3145.

Krishnamoorti, R., Vaia, R. A., \& Giannelis, E. P. (1996). Structure and dynamics of polymer-layered silicate nanocomposites. Chemistry of Materials, 8, 1728-1734.

Lan, T., Kaviratna, P. D., \& Pinnavaia, T. J. (1995). Mechanism of clay tactoid exfoliation in epoxy-clay nanocomposites. Chemistry of Materials, 7, 2144-2150. 
Li, X., Chen, J., \& Du, L. (2007). Analysis of chloro- and nitrobenzenes in water by a simple polyaniline-based solid-phase microextraction coupled with gas chromatography. Journal of Chromatography A, 1140, 21-28.

Li, X., Li, C., Chen, J., \& Sun, C. (2008). Polythiophene as a novel fiber coating for solid-phase microextraction. Journal of Chromatography A, 1198-1199, 7-13.

Li, X., Zeng, Z., Zhou, J., Gong, S., Wang, W., \& Chen, Y. (2004). Novel fiber coated with amide bridged-calix[4]arene used for solid-phase microextraction of aliphatic amines. Journal of Chromatography A, 1041, 1-9.

Lipinski, J. (2001). Automated solid phase dynamic extraction - Extraction of organics using a wall coated syringe needle. Fresenius' Journal of Analytical Chemistry, 369, 57-62.

Liu, H., Ji, L., Li, J., Liu, S., Liu, X., \& Jiang, S. (2011). Magnetron sputtering Si interlayer: A protocol to prepare solid phase microextraction coatings on metal-based fiber. Journal of Chromatography A, 1218, 2835-2840.

Liu, H., Wang, D., Ji, L., Li, J., Liu, S., Liu, X., et al. (2010). A novel TiO 2 nanotube array/Ti wire incorporated solid-phase microextraction fiber with high strength, efficiency and selectivity. Journal of Chromatography A, 1217, 1898-1903.

Lord, H., \& Pawliszyn, J. (2000). Evolution of solid-phase microextraction technology. Journal of Chromatography A, 885, 153-193.

Louch, D., Motlagh, S., \& Pawliszyn, J. (1992). Dynamics of organic compound extraction from water using liquid-coated fused silica fibers. Analytical Chemistry, 64, 1187-1199.

Love, J. C., Estroff, L. A., Kriebel, J. K., Nuzzo, R. G., \& Whitesides, G. M. (2005). Self-assembled monolayers of thiolates on metals as a form of nanotechnology. Chemical Reviews, 105, 1103-1169.

Lu, X., Chao, D., Chen, J., Zhang, W., \& Wei, Y. (2006). Preparation and characterization of inorganic/ organic hybrid nanocomposites based on Au nanoparticles and polypyrrole. Material Letters, 60 2851-2854.

MacDiarmid, A. G. (2001). "Synthetic metals": A novel role for organic polymers (Nobel lecture). Angewandte Chemie International Edition, 40, 2581-2590.

MacGillivray, B., Pawliszyn, P., Fowlie, P., \& Sagara, C. (1994). Headspace solid-phase microextraction versus purge and trap for the determination of substituted benzene compounds in water. Journal of Chromatographic Science, 32, 317-322.

Magdic, S., Boyd-Boland, A., Jinno, K., \& Pawliszyn, J. B. (1996). Analysis of organophosphorus insecticides from environmental samples using solid-phase microextraction. Journal of Chromatography A, 736, 219-228.

Mahouche-Chergui, S., Gam-Derouich, S., Mangeney, C., \& Chehimi, M. M. (2011). Aryl diazonium salts: A new class of coupling agents for bonding polymers, biomacromolecules and nanoparticles to surfaces. Chemical Society Reviews, 40, 4143-4166.

Mecerreyes, D., Alvaro, V., Cantero, I., Bengoetxea, M., Calvo, P. A., Grande, H., et al. (2002). Low surface energy conducting polypyrrole doped with a fluorinated counterion. Advanced Materials, 14, 749-752.

Mehdinia, A., \& Mousavi, M. F. (2008). Enhancing extraction rate in solid-phase microextraction by using nano-structured polyaniline coating. Journal of Separation Science, 31, 3565-3572.

Mehdinia, A., Asiabi, M., Jabbari, A., \& Kalaee, M. R. (2010). Preparation and evaluation of solid-phase microextraction fiber based on nano-structured copolymer of aniline and m-amino benzoic acid coating for the analysis of fatty acids in zooplanktons. Journal of Chromatography A, 1217, 7642-7647.

Mehdinia, A., Mohammadi, A. A., Davarani, S. S. H., \& Banitaba, M. H. (2011). Application of self-assembled monolayers in the preparation of solid-phase microextraction coatings. Chromatographia, 74, 421-427. 
Mehdinia, A., Mousavi, M. F., \& Shamsipur, M. (2006). Nano-structured lead dioxide as a novel stationary phase for solid-phase microextraction. Journal of Chromatography A, 1134, 24-31.

Mévellec, V., Roussel, S., Tessier, L., Chancolon, J., Mayne-L'Hermite, M., Deniau, G., et al. (2007). Grafting polymers on surfaces: A new powerful and versatile diazonium salt-based one-step process in aqueous media. Chemistry of Materials, 19, 6323-6330.

Minjia, H., Chao, T., Qunfang, Z., \& Guibin, J. (2004). Preparation of polyaniline coating on a stainless-steel wire using electroplating and its application to the determination of six aromatic amines using headspace solid-phase microextraction. Journal of Chromatography A, 1048, 257-262.

Mirnaghi, F. S., Chen, Y., Sidisky, L. M., \& Pawliszyn, J. (2011). Optimization of the coating procedure for a high-throughput 96-blade solid phase microextraction system coupled with LC-MS/MS for analysis of complex samples. Analytical Chemistry, 83, 6018-6025.

Mitra, S. (2003). Sample Preparation Techniques in Analytical Chemistry. Canada: John Wiley \& Sons.

Mohammadi, A., Yamini, Y., \& Alizadeh, N. (2005). Dodecylsulfate-doped polypyrrole film prepared by electrochemical fiber coating technique for headspace solid-phase microextraction of polycyclic aromatic hydrocarbons. Journal of Chromatography A, 1063, 1-8.

Mollahosseini, A., \& Noroozian, E. (2009). Polyphosphate-doped polypyrrole coated on steel fiber for the solid-phase microextraction of organochlorine pesticides in water. Analytica Chimica Acta, 638, 169-174.

Mullett, W. M., Martin, P., \& Pawliszyn, J. (2001). In-tube molecularly imprinted polymer solid-phase microextraction for the selective determination of propranolol. Analytical Chemistry, 73, 2383-2389.

Murphy, G.E. US Patent 1996, 5, 565, 622.

Musshoff, F., Lachenmeier, D.W., Kroener, L., \& Madea, B. (2003). Automated headspace solid-phase dynamic extraction for the determination of cannabinoids in hair samples. Forensic Science International, 133, 32-38.

Musshoff, F., Lachenmeier, D.W., Kroener, L., \& Madea, B. (2002). Automated headspace solid-phase dynamic extraction for the determination of amphetamines and synthetic designer drugs in hair samples. Journal of Chromatography A, 958, 231-238.

Nalwa, H. S. (1997). Handbook of Organic Conductive Molecules and Polymers. New York: Wiley.

Ohtani, A., \& Shimadzu, T. (1989). Effective doping of polymer anion during chemical polymerization of pyrrole using $\mathrm{Fe}(\mathrm{OH})_{3}$ oxidant. Bulletin of the Chemical Society of Japan, 62, 234-238.

Olszowy, P., Szultka, M., Fuchs, P., Kegler, R., Mundkowski, R., Miekisch, W., et al. (2010). New coated SPME fibers for extraction and fast HPLC determination of selected drugs in human blood. Journal of Pharmaceutical and Biomedical Analysis, 53, 1022-1027.

Olszowy, P., Szultka, M., Nowaczyk, J., \& Buszewski, B. (2011). A new way of solid-phase microextraction fibers preparation for selected antibiotic drug determination by HPLC-MS. Journal of Chromatography B: Analytical Technologies in the Biomedical and Life Sciences, 879, 2542-2548.

Pan, W., Durning, C. J., \& Turro, N. J. (1996). Kinetics of alkanethiol adsorption on gold. Langmuir, 12, 4469-4473.

Pang, L., \& Liu, J. F. (2012). Development of a solid-phase microextraction fiber by chemical binding of polymeric ionic liquid on a silica coated stainless steel wire. Journal of Chromatography $A$, 1230, 8-14.

Penton, Z. E. (1997). Sample preparation for gas chromatography with solid-phase extraction and solid-phase microextraction. Advances in Chromatography, 37, 205-236.

Picó. Y.; Fernández, M., Ruiz, M.J., \& Font, G. (2007). Current trends in solid-phase-based extraction techniques for the determination of pesticides in food and environment. Journal of Biochemical \& Biophysical Methods, 70, 117-131. 
Ponnusamy, V. K., \& Jen, J. F. (2011). A novel graphene nanosheets coated stainless steel fiber for microwave assisted headspace solid phase microextraction of organochlorine pesticides in aqueous samples followed by gas chromatography with electron capture detection. Journal of Chromatography A, 1218, 6861-6868.

Potter, D. W., \& Pawliszyn, J. (1992). Detection of substituted benzenes in water at the $\mathrm{pg} / \mathrm{ml}$ level using solid-phase microextraction and gas chromatography-ion trap mass spectrometry. Journal of Chromatography, 625, 247-255.

Potter, D. W., \& Pawliszyn, J. (1994). Rapid determination of polyaromatic hydrocarbons and polychlorinated biphenyls in water using solid-phase microextraction and GC/MS. Environmental Science \& Technology, 28, 298-305.

Prieto, A., Schrader, S., \& Moeder, M. (2010). Determination of organic priority pollutants and emerging compounds in wastewater and snow samples using multiresidue protocols on the basis of microextraction by packed sorbents coupled to large volume injection gas chromatography-mass spectrometry analysis. Journal of Chromatography A, 1217, 6002-6011.

Pron, A., \& Rannou, P. (2002). Processible conjugated polymers: From organic semiconductors to organic metals and superconductors. Progress in Polymer Science (Oxford), 27, 135-190.

Quintana, J.B., \& Rodríguez, I. (2006). Strategies for the microextraction of polar organic contaminants in water samples. Analytical \& Bioanalytical Chemistry, 2006, 384,1447-1461.

Rajabi Khorrami, A., \& Narouenezhad, E. (2011). Synthesis of molecularly imprinted monolithic fibers for solid-phase microextraction of acetaldehyde from head-space of beverages stored in PET bottles. Talanta, 86, 58-63.

Ramakrishna, S., Fujihara, K., Teo, W. E., Lim, T. C., \& Ma, Z. (2005). An Introduction to Electrospinning and Nanofibers. Singapore: World Scientific.

Rastkari, N., Ahmadkhaniha, R., \& Yunesian, M. (2009). Single-walled carbon nanotubes as an effective adsorbent in solid-phase microextraction of low level methyl tert-butyl ether, ethyl tert-butyl ether and methyl tert-amyl ether from human urine. Journal of Chromatography B: Analytical Technologies in the Biomedical and Life Sciences, 877, 1568-1574.

Rastkari, N., Ahmadkhaniha, R., Samadi, N., Shafiee, A., \& Yunesian, M. (2010). Single-walled carbon nanotubes as solid-phase microextraction adsorbent for the determination of low-level concentrations of butyltin compounds in seawater. Analytica Chimica Acta, 662, 90-96.

Rivasseau, C., \& Caude, M. (1995). Comparison of on-line SPE-HPLC and SPME-GC for the analysis of microcontaminants in water. Chromatographia, 41, 462-470.

Rout, T. K., Bandyopadhyay, N., \& Venugopalan, T. (2006). Polyphosphate coated steel sheet for superior corrosion resistance. Surface and Coatings Technology, 201, 1022-1030.

Rutledge, G. C., \& Fridrikh, S. V. (2007). Formation of fibers by electrospinning. Advanced Drug Delivery Reviews, 59, 1384-1391.

Sadki, S., Schottland, P., Brodie, N., \& Sabouraud, G. (2000). The mechanisms of pyrrole electropolymerization. Chemical Society Reviews, 29, 283-293.

Sánchez-Rojas, F., Bosch-0jeda, C., \& Cano-Pavón, J.M. (2009). A review of stir bar sorptive extraction. Chromatographia, 69, 79-94.

Santora, B. P., Gagné, M. R., Moloyt, K. G., \& Radu, N. S. (2001). Porogen and cross-linking effects on the surface area, pore volume distribution, and morphology of macroporous polymers obtained by bulk polymerization. Macromolecules, 34, 658-661.

Sarafraz-Yazdi, A., Piri-Moghadam, H., Es'Haghi, Z., \& Sepehr, S. (2010). Comparative study of the three sol-gel based solid phase microextraction fibers in extraction of BTEX from water samples using gas chromatography-flame ionization detection. Analytical Methods, 2, 746-752.

Sarafraz-Yazdi, A., Sepehr, S., Es'Haghi, Z., \& Piri-Moghadam, H. (2010). Application of sol-gel based poly(ethylene glycol)/multiwalled carbon nanotubes coated fiber for SPME of methyl tert-butyl ether in environmental water samples. Chromatographia, 72, 923-931. 
Schessler, H. M., Karpovich, D. S., \& Blanchard, G. J. (1996). Quantitating the balance between enthalpic and entropic forces in alkanethiol/gold monolayer self assembly. Journal of the American Chemical Society, 118, 9645-9651.

Shi, G., Jin, S., Xue, G., \& Li, C. (1995). A conducting polymer film stronger than aluminum. Science, 267, 994-996.

Shirakawa, H. (2001). The discovery of polyacetylene film: The dawning of an era of conducting polymers (Nobel lecture). Angewandte Chemie International Edition, 40, 2575-2580.

Shirakawa, H., Louis, E. J., MacDiarmid, A. G., Chiang, C. K., \& Heeger, A. J. (1977). Synthesis of electrically conducting organic polymers: Halogen derivatives of polyacetylene, $(\mathrm{CH}) x$. Journal of the Chemical Society, Chemical Communications, 578-580.

Shojania, S., Oleschuk, R.D., McComb, M.E., Gesser, H.D., \& Chow, A. (1999). The active and passive sampling of benzene, toluene, ethyl benzene and xylenes compounds using the inside needle capillary adsorption trap device. Talanta, 50, 193-205.

Snauwaert, P., Lazzaroni, R., Riga, J., Verbist, J. J., \& Gonbeau, D. (1990). A photoelectron spectroscopic study of the electrochemical processes in polyaniline. The Journal of Chemical Physics, 92, 2187-2193.

Soini, H.A., Bruce, K.E., Wiesler, D., David, F., Sandra, P., \& Novotny, M.V. (2005). Stir bar sorptive extraction: A new quantitative and comprehensive sampling technique for determination of chemical signal profiles from biological media. Journal of Chemical Ecology, 31, 377-392.

Svec, F. (2010). Porous polymer monoliths: Amazingly wide variety of techniques enabling their preparation. Journal of Chromatography A, 1217, 902-924.

Svec, F., \& Frechet, J. M. J. (1995). Modified poly(glycidyl methacrylate-co-ethylene dimethacrylate) continuous rod columns for preparative-scale ion-exchange chromatography of proteins. Journal of Chromatography A, 702, 89-95.

Teo, W. E., \& Ramakrishna, S. (2006). A review on electrospinning design and nanofibre assemblies. Nanotechnology, 17, R89-R106.

Tong, X., Tang, T., Feng, Z., \& Huang, B. (2002). Preparation of polymer/silica nanoscale hybrids through sol-gel method involving emulsion polymers. II. Poly(ethyl acrylate) $/ \mathrm{SiO}_{2}$. Journal of Applied Polymer Science, 86, 3532-3536.

Valor, I., Moltó, J. C., Apraiz, D., \& Font, G. (1997). Matrix effects on solid-phase microextraction of organophosphorus pesticides from water. Journal of Chromatography A, 767, 195-203.

Vuckovic, D., Cudjoe, E., Hein, D., \& Pawliszyn, J. (2008). Automation of solid-phase microextraction in high-throughput format and applications to drug analysis. Analytical Chemistry, 80 , 6870-6880.

Wang, D., Chong, S. L., \& Malik, A. (1997). Sol-gel column technology for single-step deactivation, coating, and stationary-phase immobilization in high-resolution capillary gas chromatography. Analytical Chemistry, 69, 4566-4576.

Wang, J. X., Jiang, D. Q., Gu, Z. Y., \& Yan, X. P. (2006). Multiwalled carbon nanotubes coated fibers for solid-phase microextraction of polybrominated diphenyl ethers in water and milk samples before gas chromatography with electron-capture detection. Journal of Chromatography A, 1137, 8-14.

Wang, X., Ma, Q., Li, M., Chang, C., Bai, Y., Feng, Y., et al. (2013). Automated and sensitive analysis of 28-epihomobrassinolide in Arabidopsis thaliana by on-line polymer monolith microextraction coupled to liquid chromatography-mass spectrometry. Journal of Chromatography A, 1317, 121-128.

Wang, Y., Li, Y., Zhang, J., Xu, S., Yang, S., \& Sun, C. (2009). A novel fluorinated polyaniline-based solid-phase microextraction coupled with gas chromatography for quantitative determination of polychlorinated biphenyls in water samples. Analytica Chimica Acta, 646, 78-84.

Wang, Y., Walles, M., Thomson, B., Nacson, S., \& Pawliszyn, J. (2004). Solid-phase microextraction combined with surface-enhanced laser desorption/ionization introduction for ion mobility 
spectrometry and mass spectrometry using polypyrrole coatings. Rapid Communications in Mass Spectrometry, 18, 157-162.

Wang, Y., Zhang, J., Sheng, D., \& Sun, C. (2010). Preparation and applications of perfluorinated ion doped polyaniline based solid-phase microextraction fiber. Journal of Chromatography A, 1217, 4523-4528.

Wei, Y., Tian, J., MacDiarmid, A. G., Masters, J. G., Smith, A. L., \& Li, D. (1993). Preparation and conductivities of fullerene-doped polyanilines. Journal of the Chemical Society, Chemical Communications, 603-604.

Wu, J., Yu, X., Lord, H., \& Pawliszyn, J. (2000). Solid phase microextraction of inorganic anions based on polypyrrole film. The Analyst, 125, 391-394.

Xu, Q., Wu, S. Y., Wang, M., Yin, X. Y., Wen, Z. Y., Ge, W. N., et al. (2010). Electrospun nylon6 nanofibrous membrane as SPE adsorbent for the enrichment and determination of three estrogens in environmental water samples. Chromatographia, 71, 487-492.

Yoshimatsu, K., Ye, L., Lindberg, J., \& Chronakis, I. S. (2008). Selective molecular adsorption using electrospun nanofiber affinity membranes. Biosensors and Bioelectronics, 23, 1208-1215.

Yu, J., Dong, L., Wu, C., Wu, L., \& Xing, J. (2002). Hydroxyfullerene as a novel coating for solid-phase microextraction fiber with sol-gel technology. Journal of Chromatography A, 978, 37-48.

Yu, J., Wu, C., \& Xing, J. (2004). Development of new solid-phase microextraction fibers by sol-gel technology for the determination of organophosphorus pesticide multiresidues in food. Journal of Chromatography A, 1036, 101-111.

Zewe, J. W., Steach, J. K., \& Olesik, S. V. (2010). Electrospun fibers for solid-phase microextraction. Analytical Chemistry, 82, 5341-5348.

Zhang, M., Wei, F., Zhang, Y.-F., Nie, J., Feng, Y. Q. (2006). Novel polymer monolith microextraction using a poly(methacrylic acid-ethylene glycol dimethacrylate) monolith and its application to simultaneous analysis of several angiotensin II receptor antagonists in human urine by capillary zone electrophoresis. Journal of Chromatography A, 1102, 294-301.

Zhang, W., \& Chen, Z. (2013). Mussel inspired polydopamine functionalized poly(ether ether ketone) tube for online solid-phase microextraction-high performance liquid chromatography and its application in analysis of protoberberine alkaloids in rat plasma. Journal of Chromatography $A$, 1278, 29-36.

Zhang, Z., \& Pawliszyn, J. (1993). Headspace solid-phase microextraction. Analytical Chemistry, 65, 1843-1852.

Zhang, Z., Yang, M. J., \& Pawliszyn, J. (1994). Solid-phase microextraction. Analytical Chemistry, 66, 844A-853A.

Zheng, M. M., Ruan, G. D., \& Feng, Y. Q. (2009). Evaluating polymer monolith in-tube solid-phase microextraction coupled to liquid chromatography/quadrupole time-of-flight mass spectrometry for reliable quantification and confirmation of quinolone antibacterials in edible animal food. Journal of Chromatography A, 1216, 7510-7519.

Zheng, M. M., Wang, S. T., Hu, W. K., \& Feng, Y. Q. (2010). In-tube solid-phase microextraction based on hybrid silica monolith coupled to liquid chromatography-mass spectrometry for automated analysis of ten antidepressants in human urine and plasma. Journal of Chromatography $A, 1217$, 7493-7501.

Zhou, J., Yang, F., Cha, D., Zeng, Z., \& Xu, Y. (2007). Headspace solid-phase microextraction with novel sol-gel permethylated- $\beta$-cyclodextrin/hydroxyl-termination silicone oil fiber for determination of polybrominated diphenyl ethers by gas chromatography-mass spectrometry in soil. Talanta, $73,870-877$.

Zhou, S., Wu, L., Sun, J., \& Shen, W. (2002). The change of the properties of acrylic-based polyurethane via addition of nano-silica. Progress in Organic Coatings, 45, 33-42.

Zou, J., Song, X., Ji, J., Xu, W., Chen, J., Jiang, Y., et al. (2011). Polypyrrole/graphene composite-coated fiber for the solid-phase microextraction of phenols. Journal of Separation Science, 34, 2765-2772. 